\title{
Protein Kinase C Regulates ErbB3 Turnover
}

\author{
Markus Dietrich ${ }^{1,2}$, Muhammad Salman Malik ${ }^{1,3}$, Marianne Skeie ${ }^{2}$, Vibeke Bertelsen $^{1,4}$ and \\ Espen Stang ${ }^{1, *}$ \\ ${ }^{1}$ Department of Pathology, Oslo University Hospital, Oslo, Norway \\ ${ }^{2}$ Institute of Clinical Medicine, University of Oslo, Oslo, Norway \\ ${ }^{3}$ Current address: Department of Food Safety and Infection Biology, \\ Norwegian University of Life Sciences, Oslo, Norway \\ ${ }^{4}$ Current address: Department of Forensic Medicine, Oslo University Hospital, Oslo, Norway
}

\begin{abstract}
*Corresponding author: Espen Stang, Department of Pathology, Oslo University Hospital, Post box 4950 Nydalen, 0424 Oslo, Norway.

Tel: 47-23071483. E-mail: espsta@rr-research.no
\end{abstract}

Abbreviations: CHX, cycloheximide; DAG, diacylglycerol; EEA1, early endosomal antigen 1; HRG, heregulin; LAMP1, lysosomal-associated membrane protein 1; PMA, phorbol 12-myristate 13-acetate; RTK, receptor tyrosine kinase 


\begin{abstract}
ErbB3, which belongs to the epidermal growth factor receptor (EGFR) or ErbB family of receptor tyrosine kinases, is involved in progression of several human cancers and a tight regulation of its expression is crucial. An important mechanism for regulation of ErbB proteins is endocytosis and we recently showed that ErbB3, contrary to other ErbB proteins, like EGFR and ErbB2, is constitutively internalized and degraded. Several studies show that protein kinase C (PKC) can regulate the activation, localization and stability of EGFR and ErbB2. Activation of PKC causes their down-regulation from the plasma membrane, but instead of being degraded the receptors accumulate in an endosomal recycling compartment. Since little is known about possible connections between ErbB3 and PKC, we have in the present study investigated effects PKC activity has on ErbB3 stability and intracellular trafficking. While PKC inhibition tends to increase ErbB3 degradation, activation of PKC causes ErbB3 stabilization. The stabilization was not due to inhibited internalization, on the contrary we find that expression of ErbB3 at the plasma membrane is reduced upon PMA-induced PKC activation. However, while endocytosed ErbB3 under normal conditions and upon PKC inhibition is found in early endosomal antigen 1 (EEA1) positive early endosomes and lysosomal-associated membrane protein 1 (LAMP1) positive late endosomes/lysosomes, indicating that it follows the classic degradative pathway, ErbB3 localizes to EEA1 and LAMP1 negative compartments upon PMA-induced activation of PKC. Altogether this shows that PKC regulates the stability of ErbB3, and knockdown experiments show that $\mathrm{PKC} \delta$ is essential in this process. A likely explanation is that $\mathrm{PKC}$ regulates endosomal sorting of ErbB3 and that activated PKC sorts ErbB3 away from the degradative pathway.
\end{abstract}

Keywords: ErbB3; protein kinase C (PKC); phorbol 12-myristate 13-acetate (PMA); endosomal sorting; receptor internalization; receptor recycling 


\section{INTRODUCTION}

The ErbB family of receptor tyrosine kinases (RTKs) consists of four closely related members: epidermal growth factor receptor (EGFR, or ErbB1), ErbB2/HER2, ErbB3/HER3 and ErbB4/HER4. Signaling down-stream of the ErbB proteins depends among others, on ligand specificity, dimerization status, kinase activity and subcellular localization (Arteaga and Engelman 2014, Roskoski 2014).

ErbB3 has a relatively short protein half-life of around 2.5-3.5 hours (Waterman, Sabanai et al. 1998) and the half-life decreases remarkably upon ligand binding (around 0.5 hours) (Cao, Wu et al. 2007). It has been reported that an endoplasmic reticulum-localized quantity control partially regulates the ligand-independent steady-state expression of ErbB3 (Fry, Simion et al. 2011). However, in general the half-life of ErbB proteins is considered to be regulated by endocytosis (Sorkin and Goh 2009), and although the turnover of ErbB3 increases upon ligand binding, we recently demonstrated that ErbB3 is constitutively endocytosed in absence of ligand and independent of its phosphorylation (Sak, Breen et al. 2012, Fosdahl, Dietrich et al. 2017).

The protein kinase $\mathrm{C}$ (PKC) family consists of protein kinases causing serine- and/or threoninephosphorylation of their clients. The family is sub classified into conventional or classical PKCs which require diacylglycerol (DAG), calcium ions $\left(\mathrm{Ca}^{2+}\right)$ and phospholipids for activation, novel PKCs which need DAG but not $\mathrm{Ca}^{2+}$, and atypical PKCs which require neither DAG nor $\mathrm{Ca}^{2+}$ but phosphatidylserine for full activation. Agonist binding to several different RTKs or G-protein coupled receptors (GPCRs) can activate PKCs. Upon activation of RTKs or GPCRs phospholipase C (PLC) isoforms hydrolyze phosphatidylinositol 4,5-bisphosphate (PIP2) to DAG and inositol 1,4,5-triphosphate (IP3), which in turn leads to a release of $\mathrm{Ca}^{2+}$ from intracellular storages (Rozengurt 2007). After activation PKCs translocate from the cytosol to the plasma membrane, allowing them to phosphorylate their substrates (Mosior and Newton 1996).

The tumor promoting phorbol ester phorbol 12-myristate 13-acetate (PMA, also known as TPA) is known to be a potent PKC activator. PMA can directly activate conventional and novel PKCs by mimicking and thereby utilizing the same activation mechanism as DAG. It has previously been shown that PMA-induced activation of PKCs can cause down-regulation of EGFR and ErbB2 from the plasma membrane without leading to their subsequent degradation. The receptors are instead routed to and, to varying degree, sequestered in endosomes referred to as the endocytic recycling compartment (ERC) or the pericentrion (Bao, Alroy et al. 2000, Llado, Timpson et al. 2008, Idkowiak-Baldys, Baldys et al. 2009, Liu, Idkowiak-Baldys et al. 2013, Bailey, Luan et al. 2014, Dietrich, Malik et al. 2018). Contrary to what is reported for EGFR (Bao, Alroy et al. 2000), down-regulation of ErbB2 appears to depend on its kinase activity (Bailey, Luan et al. 2014). For EGFR the effect depends on phosphorylation of threonine 654 (Thr-654) which is the major phosphorylation site for PKC on EGFR (Hunter, Ling et al. 1984, Lund, Lazar et al. 1990, Bao, Alroy et al. 2000, Liu, Idkowiak-Baldys et al. 2013). Likewise, the PKC activator phorbol dibutyrate (PdBU) has been shown to induce phosphorylation of the corresponding Thr-686 in ErbB2 (Ouyang, Gulliford et al. 1996, Ouyang, Gulliford et al. 1998). Little is known with respect to PMA-induced activation of PKCs and effects on ErbB3. Importantly, the juxtamembrane domain of ErbB3 does not contain a threonine in the position corresponding to Thr-654 in EGFR or Thr-686 in ErbB2. In ErbB3 the corresponding amino acid 
is alanine (Ouyang, Gulliford et al. 1998), but it has been shown that PMA can stimulate tyrosine phosphorylation of both ErbB2 and ErbB3 in a PKC dependent manner (Emkey and Kahn 1997). In the present study we have investigated in more detail the effects PKCs have on ErbB3. Surprisingly, both activation and inhibition of PKC caused reduced expression of ErbB3 at the plasma membrane. However, while inhibition of PKC increased the ligand-independent ErbB3 degradation, PMA-mediated PKC activation induced stabilization of ErbB3. Furthermore, PKC activation altered the intracellular trafficking of ErbB3 away from early and late endosomes. siRNA-induced knockdown of various PKC isoforms showed that PKC $\delta$ is essential for the PMA-induced stabilization of ErbB3.

\section{MATERIALS AND METHODS}

\section{Reagents and Antibodies}

Mouse anti-ErbB3 (Ab-6), Alexa Fluor 555-conjugated donkey anti-mouse IgG and Alexa Fluor 647-conjugated donkey anti-goat IgG antibodies were from Thermo Fisher Scientific (Waltham, MA, USA). Rabbit anti-ErbB3 (C-17), mouse anti-ubiquitin, mouse anti-phosphotyrosine and goat anti-early endosome antigen 1 (EEA1) (N-19) antibodies were from Santa Cruz Biotechnology (Santa Cruz, CA, USA). Rabbit anti-ErbB3 (D22C5) was from Cell Signaling Technology, Inc. (Danvers, MA, USA). Rabbit anti- $\beta$-tubulin, rabbit anti-LAMP1, rabbit anti$\mathrm{PKC} \alpha$ and rabbit anti-PKC $\eta$ were from Abcam (Cambridge, UK). Mouse anti-PKC $\delta$ antibody was from BD Biosciences (San Jose, CA, USA). Unconjugated, as well as PE- and APCconjugated, mouse anti-ErbB3 (1B4C3) was from BioLegend (San Diego, CA, USA). Peroxidase-conjugated donkey anti-rabbit and anti-mouse IgG antibodies and Alexa Fluor 488conjugated donkey anti-rabbit IgG were from Jackson ImmunoResearch Laboratories (West Grove, PA, USA). Ro 31-8220 and Gö 6976 were from Merck Millipore (Darmstadt, Germany). Heregulin $\beta 1$-extracellular domain (HRG) was from R\&D systems Inc. (Minneapolis, MN, USA). Unless noted, all other materials and chemicals were from Sigma-Aldrich (St. Louis, MO, USA).

\section{Cell Culture and Treatment}

MCF-7 cells were grown in DMEM with Ultraglutamine I and $4.5 \mathrm{~g} / \mathrm{l}$ Glucose (Lonza, Basel, Switzerland). A mixture of 50 units of Potassium Penicillin and $50 \mu \mathrm{g}$ of Streptomycin Sulfate per $1 \mathrm{ml}$ of DMEM and 10\% (v/v) fetal bovine serum were routinely used. PMA, Ro 31-8220 and Gö 6976 were dissolved in DMSO and therefore DMSO was used as vehicle control in all experiments where PMA, Ro 31-8220 or Gö 6976 were present.

siRNA transfection of cells

For knockdown experiments, cells were transfected with short interfering RNA (siRNA) twice with a $48 \mathrm{~h}$ interval using Lipofectamine ${ }^{\circledR}$ RNAiMax (Thermo Fisher Scientific). Cells were used for experiments $48 \mathrm{~h}$ after the second transfection. The transfection was performed according to the manufacturer's protocol using a final concentration of $50 \mathrm{nM}$ siRNA. For knockdown of all PKCs, PKC siRNA (h) (sc-29449) from Santa Cruz Biotechnology was used. SMARTpool of ON-TARGETplus PRKCD siRNA (L-003524-00) and On-TARGETplus Non-targeting siRNA \#2 (D-001810-02) were from Dharmacon (Horizon Discovery, Cambridge, UK). 


\section{Immunoblotting}

Cells were lysed in SDS (sodium-dodecyl sulfate) lysis buffer (Szymanska, Fosdahl et al. 2016) supplemented with $10 \mu \mathrm{g} / \mathrm{ml}$ protease inhibitor and phosphatase inhibitor cocktails and subjected to homogenization using a QIAshredder column (QIAGEN, Valencia, CA, USA). Sample buffer (Szymanska, Fosdahl et al. 2016) was added to the lysates before incubation for $10 \mathrm{~min}$ at $95^{\circ} \mathrm{C}$. Proteins were separated by SDS gel electrophoresis and protein blotting using $10 \%$ MiniPROTEAN®TGX ${ }^{\mathrm{TM}}$ Precast Gels and Trans-Blot ${ }^{\circledR}$ Turbo ${ }^{\mathrm{TM}}$ Transfer System (Bio-Rad Laboratories, Hercules, CA, USA). The membranes were incubated with primary and secondary antibodies diluted in $1 \%$ Blotting-Grade Blocker (Bio-Rad Laboratories) for 2 hours at room temperature or over night at $4^{\circ} \mathrm{C}$ before detection of proteins using Super Signal West Dura Extended Duration Substrate from Thermo Fisher Scientific (Waltham, MA USA) and visualization using the ChemiDoc MP System (Bio-Rad Laboratories). Image Lab Software version 4.0.1 build 6 (Bio-Rad Laboratories) and Adobe Photoshop (Adobe Systems, San José, CA, USA) were used for image processing.

\section{Flow Cytometry}

Cells were seeded one day prior to experiments in 6-well plates. Following treatment, cells were rinsed 3x with PBS and detached with trypsin/EDTA. Trypsinization was stopped by addition of trypsin inhibitor. Cell suspensions were transferred to 96 -well plates and washed $2 \mathrm{x}$ with PBS. Cells were stained $30 \mathrm{~min}$ on ice with fixable viability dye (eBioscience Inc., San Diego, CA, USA) for dead-live discrimination and afterwards washed $2 \mathrm{x}$ with FACS buffer $(0.5 \%$ fetal bovine serum $/ 0.1 \%$ EDTA $\mathrm{pH}=8$ in PBS). Cells were fixed at room temperature in $4 \%$ PFA for 10 min. For ErbB3 surface staining, cells were incubated 15 min on ice with either PE- or APCconjugated anti-ErbB3 antibody and washed 2x with FACS buffer. Cells stained with PEconjugated antibody were run on LSR II flow cytometer and cells stained with APC-conjugated antibody were run on LSR Fortessa cell analyzer both from BD Biosciences. Results were analyzed with FlowJo software version 10.1 (FlowJo, LLC, Ashland, OR, USA) and GraphPad Prism 6 (GraphPad Software, Inc., La Jolla, CA, USA).

\section{ErbB3 internalization assay}

Cells were incubated with a mouse anti-ErbB3 antibody (1B4C3) recognizing the extracellular part of the receptor, in MEM with $0.1 \%$ bovine serum albumin for $30 \mathrm{~min}$ at $18^{\circ} \mathrm{C}$ and chased in $\mathrm{MEM}+0.1 \%$ bovine serum albumin for $30 \mathrm{~min}$ at $37^{\circ} \mathrm{C}$. Before fixation in $4 \%$ PFA cells were washed for $2 \mathrm{~min}$ in low $\mathrm{pH}$ buffer $(0.5 \%$ acetic acid, $0.5 \mathrm{M} \mathrm{NaCl}, \mathrm{pH}=2.8)$ to remove surface bound antibodies. Upon fixation, quenching and permeabilization with $50 \mathrm{mM} \mathrm{NH}_{4} \mathrm{Cl}$ and $0.01 \%$ Triton X-100, internalized anti-ErbB3 antibodies were detected using an Alexa Fluor 555conjugated anti-mouse antibody. EEA1 antibodies were detected with an Alexa Fluor 647conjugated anti-goat antibody and LAMP1 antibodies were detected with an Alexa Fluor 488conjugated anti-rabbit antibody.

\section{Imaging and Image Analysis}

Laser scanning confocal microscopy was done on an Olympus Fluoview 1000 (BX61W1) system with 405, 488, 543 and 633nm laser lines and an UPlanSApo 60x oil objective with a numerical aperture of 1.35 (Olympus). Images were acquired with the FV10-ASW-v.42 software and 
montages were assembled in ImageJ (Version2.0.0-rc41/1.50d; Java 1.6.0_24 [64-bit]) from National Institutes of Health, USA.

To calculate co-localization of ErbB3 and EEA1, we performed a quantitative image analysis to determine the amount of anti-ErbB3 antibodies co-localizing with EEA1, using ImageJ. For each condition we analyzed 23 pictures acquired as mentioned above from six different experiments on cells treated with PMA or the respective amount of DMSO as a control condition. For every single picture we created 8-bit binary pictures for each channel (ErbB3 and EEA1) and used an auto threshold filter (MaxEntropy) where bright spots representing either EEA1 or ErbB3 where enhanced and represented as white dots while all background was set to black and thereby no longer detectable. By the use of the plugin Binary Feature Extractor (The BioVoxxel Image Processing and Analysis Toolbox, Brocher, 2015) white spots in both single images were automatically counted and by counting of white spots overlapping at least $25 \%$ in both pictures, we got a count of internalized ErbB3 antibodies, EEA1 positive compartments, and ErbB3 antibodies co-localizing with EEA1. We then calculated mean, standard deviation and standard error for mean of the numbers representing internalized ErbB3 antibodies co-localizing with EEA1 and tested for statistical significance as described below.

\section{Statistical Data Analysis}

Statistical analysis of western blots and flow cytometry was performed using GraphPad Prism 6. Data analyzed were the results of at least three independent experiments. Mean \pm SD was calculated and statistical significance was determined by using one unpaired t-test per row.

Image $\mathrm{J}$ data was analyzed by GraphPad Prism 6 using unpaired $t$-test with Welch's correction. Significance level were set to n.s. non-significant, * $\mathrm{p}<0.05$, ** $\mathrm{p}<0.01$, *** $\mathrm{p}<0.001$, $* * * * \mathrm{p}<0.0001$.

\section{Immunoprecipitation}

To study ubiquitination of ErbB3, cells were lysed in preheated $\left(100^{\circ} \mathrm{C}\right) 1 \%$ SDS in PBS, incubated at $100^{\circ} \mathrm{C}$ for $5 \mathrm{~min}$ and homogenized using a QIA-shredder column (QIAGEN). The lysates were added to protein A-coupled magnetic beads $(2.8 \mu \mathrm{M})$ (Thermo Fisher Scientific) precoupled to mouse anti-ErbB3 (Ab-6) antibody in $0.1 \mathrm{M}$ phosphate buffer, $\mathrm{pH} 8$, with $0.05 \%$ Triton X-100 at room temperature for 1 hour. The beads were resuspended in $2 \mathrm{x}$ immunoprecipitation (IP) buffer (2\% (v/v) Triton X-100, 0.5\% (w/v) sodium deoxycholate, $1 \%$ (w/v) bovine serum albumin, $2 \mathrm{mM}$ EDTA, $40 \mathrm{mM} \mathrm{NaF}, 6 \mathrm{mM}$ NEM, 1:100 (v/v) protease inhibitor and phosphatase inhibitor cocktail). Antibody-coupled magnetic beads and cell lysates were gently mixed for 1 hour at $4^{\circ} \mathrm{C}$. The beads were then washed four times with $1 \mathrm{x}$ IP buffer (50\% 2x IP buffer $+50 \%$ SDS [1\%] in PBS), once in 1:10 diluted PBS and then eluted in 2x sample buffer at $95^{\circ} \mathrm{C}$ for 5 min. Immunoprecipitated ErbB3 was analyzed by immunoblotting with antibodies to ErbB3 and ubiquitin.

\section{RESULTS}

\section{Activation of PKC causes stabilization of ErbB3}

Turnover of ErbB proteins is to a large extent regulated by their internalization followed by either recycling to the plasma membrane, or sorting via early endosomes to late endosomes and 
lysosomes for degradation. Activation of PKCs has, however, been shown to inhibit the degradation of EGFR and ErbB2 (Bao, Alroy et al. 2000, Llado, Timpson et al. 2008, IdkowiakBaldys, Baldys et al. 2009, Liu, Idkowiak-Baldys et al. 2013, Bailey, Luan et al. 2014, Dietrich, Malik et al. 2018). Since we and others have previously shown that ErbB3 is constitutively internalized and degraded with a short half-life (Waterman, Sabanai et al. 1998, Cao, Wu et al. 2007, Sak, Breen et al. 2012, Fosdahl, Dietrich et al. 2017), we first investigated the effect prolonged PKC-activation had on the total ErbB3 protein level. Incubation of MCF-7 cells with PMA alone, which activates both conventional and novel PKCs, showed no clear effect on the total ErbB3 level (Figure 1A). When cells were treated with cycloheximide (CHX), which inhibits protein synthesis, immunoblotting demonstrated a rapid decrease in the ErbB3 level (Figure 1A and B), confirming a constitutive degradation of ErbB3. However, when PMA was combined with $\mathrm{CHX}$, the constitutive degradation of ErbB3 was strongly reduced and even after 12 hours ErbB3 was clearly present (Figure 1B and C) indicating a PKC-induced stabilization of ErbB3.

It is known that prolonged treatment with phorbol esters down-regulates both conventional and novel PKCs due to proteolysis of activated PKC isozymes (Parker, Bosca et al. 1995). PMAinduced degradation of PKCs was thus included as control of PMA activity. We analyzed the expression level of $\mathrm{PKC} \alpha$ and $\mathrm{PKC} \delta$, a conventional and a novel PKC, respectively. While PKC $\delta$ was rapidly degraded, degradation of $\mathrm{PKC} \alpha$ was slower but clearly detectable upon four hour incubation with PMA (Figure 1A-B).

To further investigate the role of PKCs in ErbB3 turnover, we initially used the broad spectrum PKC inhibitor Ro 31-8220, which has been shown to inhibit conventional PKCs like PKC $\alpha, \beta I$, $\beta \mathrm{II}$ and $\gamma$, and the novel PKC $\varepsilon$ (Nixon, Bishop et al. 1992, Wilkinson, Parker et al. 1993). The ErbB3 stabilization we observed when PMA was combined with CHX was inhibited by Ro 318220, and incubation with Ro 31-8220 in combination with CHX caused, if anything, a slight increase in ErbB3 degradation (Figure 2 A and B). As expected Ro 31-8220 inhibited PMAinduced degradation of the conventional PKC $\alpha$ but not the novel PKC $\delta$ (Figure 2A).

\section{PKC Regulates the Plasma Membrane Level of ErbB3}

Several different mechanisms may be the reason behind the observed PMA-induced stabilization of ErbB3. Since ErbB3 is constitutively internalized and degraded (Sak, Breen et al. 2012), inhibited internalization could be one mechanism. The expression of ErbB3 has also been shown to be regulated by an endoplasmic reticulum-localized quantity control (Fry, Simion et al. 2011), and inhibition of this would show up as a stabilization. Inhibition of either of these mechanisms can be expected to cause an increase in the level of ErbB3 at the plasma membrane. Activation of PKCs has on the other hand been shown to down-regulate EGFR and ErbB2 from the plasma membrane, without causing their subsequent degradation (Bao, Alroy et al. 2000, Llado, Timpson et al. 2008, Idkowiak-Baldys, Baldys et al. 2009, Liu, Idkowiak-Baldys et al. 2013, Bailey, Luan et al. 2014, Dietrich, Malik et al. 2018). Due to this we investigated the effect a prolonged PKC activation had on plasma membrane localized ErbB3. When MCF-7 cells were incubated with PMA, flow cytometric analysis showed that the plasma membrane expression of ErbB3 was indeed decreased, especially within the first two hours. Upon longer incubation times, the effect diminished and was partially reversed (Figure 3A). Treatment with CHX caused the same initial decrease in ErbB3 plasma membrane expression as PMA, but while the effect of PMA leveled off 
after two hours, the effect of CHX continued (Figure 3A). The seemingly similar initial effect of CHX and PMA could suggest that PMA has effect on ErbB3 synthesis. The PMA induced stabilization of total ErbB3 does however make this unlikely, and when cells were treated with CHX and PMA in combination, the initial effect was increased (Figure 3A), supporting that PMA and $\mathrm{CHX}$ affect ErbB3 by different mechanisms. Upon four-hour incubation the ErbB3 plasma membrane level was equal in cells treated with CHX only or with the PMA CHX combination, indicating that the effect of PMA on plasma membrane expression of ErbB3 is transient. The exact reason for this transient effect is unclear, but a likely explanation is the PMA-induced gradual degradation of both conventional and novel PKCs (Figure 1 A-B).

To further investigate the role of PKCs in regulation of the ErbB3 plasma membrane level, we used Ro 31-8220. Treating the cells with the inhibitor alone did not show any effect on ErbB3 plasma membrane expression (Figure 3B). When cells were incubated with Ro 31-8220 in combination with CHX, the PKC inhibitor initially showed an effect similar to PMA combined with CHX (Figure 3B). However, in line with a negative effect of Ro 31-8220 on ErbB3 stability (Figure 2A), prolonged incubation with Ro 31-8220 in combination with CHX caused an increased down-regulation of ErbB3 from the plasma membrane, and after six hours the ErbB3 level was far below the values seen upon incubation with CHX only or the combination of PMA and CHX. When PMA was combined with Ro 31-8220 and CHX, the effect was mainly as with the Ro 31-8220 CHX combination. Altogether the data show that PKCs are important regulators of ErbB3 plasma membrane expression and although Ro 31-8220 alone had no detectable effect, the results with Ro 31-8220 in combination with CHX indicate that a certain basal PKC activity is necessary to sustain a constant level of ErbB3 at the plasma membrane.

\section{PKC Regulates Intracellular Sorting of ErbB3}

Since activation of PKCs stabilized the total expression of ErbB3 but decreased the level of ErbB3 at the plasma membrane, we next investigated the effect PKC activation had on intracellular localization and sorting of ErbB3. We concentrated on internalization and endosomal sorting of ErbB3. To study this, we used an antibody-based internalization assay combined with confocal laser scanning microscopy as described previously (Sak, Breen et al. 2012). Internalization of antibody-ErbB3 complexes appeared to occur to a similar extent under control conditions and upon PMA induced PKC activation. However, while antibodies internalized for 30 min in control cells to a large extent localized to EEA1-positive endosomes, co-localization with EEA1 was reduced upon treatment with PMA (Figure 4A, for higher magnification see Supplementary Figure 1). By quantitative image analysis and calculation of the ratio of antiErbB3 antibodies co-localizing with EEA1 to total internalized anti-ErbB3 antibodies, statistical analysis showed that under normal conditions approximately $30 \%$ of internalized ErbB3 localized to EEA1-positive endosomes, while after PMA treatment this co-localization was reduced to $15 \%$ (Figure 4B).

Furthermore, we checked if anti-ErbB3 antibodies were transported to LAMP1 positive compartments. While the antibody-positive compartments in control cells partially were LAMP1positive, the compartments in PMA-treated cells were mainly LAMP1-negative, indicating that PKC activation prevents sorting of ErbB3 towards the degradative pathway (Figure 5A). This is in support to the observation that activation of PKC does not induce ErbB3 degradation, but 
rather ErbB3 stabilization. Due to the very low level of co-localization, a quantitative image analysis was not performed.

When incubating cells with Ro 31-8220 we could not determine any clear localization differences between DMSO (see Figure 4A) and Ro 31-8220 treated cells (Figure 5B), indicating that PKC inhibition increases ErbB3 degradation by routing ErbB3 along a classic degradative pathway.

\section{Inhibited Recycling is not Sufficient for Stabilization of ErbB3}

Based on our current data and on previous studies of EGFR and ErbB2, it is likely that activation of PKC causes accumulation and stabilization of ErbB3 in some kind of recycling compartment. We checked for co-localization of ErbB3 with transferrin which is known to recycle rapidly upon internalization. ErbB3 and transferrin did, however, not show any extensive co-localization neither under control conditions, nor upon treatment with PMA (data not shown), excluding that ErbB3 is sorted to transferrin-containing recycling endosomes upon PKC activation.

To test if inhibited recycling is sufficient to decrease the plasma membrane expression of ErbB3, we treated cells with the recycling inhibitor monensin. Incubation with monensin in combination with CHX for two hours caused the same down-regulation of ErbB3 from the plasma membrane as incubation with PMA combined with CHX. However, upon longer incubation (4-6 hours), the monensin CHX combination decreased the plasma membrane levels even more than the PMA CHX combination or CHX alone (Figure 6A). This suggests that relatively large amounts of ErbB3 under normal conditions are internalized and rapidly recycled. When analyzing ErbB3 degradation, we found no stabilization of ErbB3 upon incubation with monensin (Figure 6B and C), and confocal microscopy analysis showed that ErbB3 localized to LAMP1 positive compartments after incubation with monensin for two hours (Figure 6D).

This shows that the monensin-inhibited recycling is not sufficient to inhibit ligand-independent degradation of ErbB3. Surprisingly, when monensin was combined with PMA, degradation of ErbB3 was inhibited (Figure 6B and C) and the plasma membrane down-regulation was exactly the same as for PMA alone (Figure 6A). A likely explanation is that monensin and PMA cause different endosomal sorting of ErbB3, and that while monensin inhibits recycling but allows sorting to late endosomes and lysosomes, activation of PKCs sorts ErbB3 away from the degradative pathway at an early stage (as shown in Figure 4A).

\section{Activation of PKC Does not Inhibit Heregulin-Induced Degradation of ErbB3}

We and others have previously shown that stimulation with heregulin (HRG) leads to a rapid degradation of ErbB3 (Warren, Kani et al. 2006, Sak, Breen et al. 2012, Fosdahl, Dietrich et al. 2017). To test the influence of PKC activation on HRG-induced trafficking of ErbB3, we first investigated effects of HRG on ErbB3 plasma membrane expression. HRG induced a rapid downregulation of ErbB3 from the plasma membrane. Upon one hour incubation with a combination of HRG and CHX the ErbB3 plasma membrane level was far below levels found in cells treated with CHX only or with a combination of CHX and PMA (Figure 7A). We could, however, not detect any clear difference between HRG only and HRG plus PMA stimulated cells (Figure 7A). Furthermore, we tested if PKC activation can inhibit HRG-induced ErbB3 degradation. There was, however, no observable effect of PMA on HRG-induced degradation of ErbB3 (Figure 7B and C). 


\section{Activation of PKC Does not Induce Tyrosine Phosphorylation or Ubiquitination of ErbB3}

We and others have previously shown that ubiquitination of EGFR and ErbB2 functions as a signal both for internalization and for sorting to late endosomes and lysosomes for degradation (Bertelsen, Sak et al. 2011, Vuong, Berger et al. 2013). HRG is known to induce tyrosine phosphorylation (Pinkas-Kramarski, Shelly et al. 1996) and ubiquitination of ErbB3 (Cao, Wu et al. 2007), and ubiquitination has been shown to regulate both constitutive and HRG-induced degradation of ErbB3 (Cao, Wu et al. 2007, Szymanska, Fosdahl et al. 2016, Fosdahl, Dietrich et al. 2017). As shown in Figure 8, ErbB3 was tyrosine phosphorylated and ubiquitinated upon incubation with HRG, but not with PMA. Importantly the experiments also showed that although incubation with PMA to some degree reduced the HRG-induced ubiquitination of ErbB3, the ubiquitination was not blocked. This may explain why PMA cannot compensate for HRGinduced internalization and degradation of ErbB3.

\section{PKC $\delta$ is essential for PMA-induced stabilization of ErbB3}

In the experiments above, Ro 31-8220 was used to inhibit PKC activity. Ro 31-8220 was originally reported to specifically inhibit conventional PKCs (Nixon, Bishop et al. 1992), but was later found to inhibit also the novel PKCE (Wilkinson, Parker et al. 1993), as well as kinases like MSK1, MAPKAPK1, S6K1 and GSK3 (reviewed in Davies, Reddy et al. 2000, Goekjian and Jirousek 2001). Effects of Ro 31-8220 on the novel PKCs, PKC $8, \mathrm{PKC} \eta$ and PKC $\theta$, are to our knowledge not reported. We next used the PKC inhibitor Gö 6976 which inhibits PKC $\alpha$ and $\mathrm{PKC} \beta$, but has minimal effects on $\mathrm{PKC} \varepsilon, \mathrm{PKC} \delta$ and $\mathrm{PKC} \eta$ (Goekjian and Jirousek 2001). Surprisingly, Gö 6976 had no significant effect on PMA-induced ErbB3 stabilization (Figure 9). Although we cannot exclude PKC $\gamma$, this suggests that, contrary to what was expected based on Ro 31-8220, conventional PKCs are not responsible for the observed effects. To verify that PMAinduced stabilization of ErbB3 depends on PKC activity, we knocked down PKCs using a pan PKC siRNA. As shown in Figure 10, this efficiently inhibited the ErbB3 stabilization. To identify the isoform(s) involved, we then knocked down PKC $\alpha, \mathrm{PKC} \beta, \mathrm{PKC} \delta, \mathrm{PKC} \gamma, \mathrm{PKC} \varepsilon$ and $\mathrm{PKC} \eta$ one by one. While knockdown of PKC $\delta$ had a significant effect (Figure 11), knockdown of the other isoforms had no reproducible effect (data not shown). It should be noted that knockdown of $\mathrm{PKC} \gamma$ was not verified since the antibodies tested did not significantly confirm its expression. We also tested various combinations of siRNAs two by two without observing any significant additional effects (data not shown). This shows that PKC $\delta$ is essential for PMA-induced stabilization of ErbB3, but since we find an effect of Ro 31-8220, we cannot exclude that other isoforms also contribute.

\section{DISCUSSION}

In addition to constitutive and ligand-induced turnover, expression and localization of ErbB proteins are regulated through trans-activation by other receptors, both RTKs and GPCRs, and by other kinases like the PKCs. It has been shown for both EGFR (Bao, Alroy et al. 2000, Llado, Timpson et al. 2008, Liu, Idkowiak-Baldys et al. 2013) and ErbB2 (Bailey, Luan et al. 2014, Dietrich, Malik et al. 2018) that PKC activation can induce their down-regulation from the plasma membrane and alter their intracellular sorting. In the current study we investigated and compared the effects of the PKC activator PMA, the PKC inhibitors Ro 31-8220 and Gö 6976, and siRNA-induced knockdown of PKCs, on ErbB3 localization and stability. At the same time 
as PMA induced ErbB3 stabilization, it induced a rapid decrease in the level of ErbB3 expressed at the plasma membrane. We cannot definitely determine whether the decreased plasma membrane level is caused by induced internalization, inhibited recycling, or by inhibited transport of newly synthesized ErbB3 along the secretory pathway. However, as will be discussed, we favor the notion that PKC regulates endosomal sorting of ErbB3 and that PMA-induced activation of PKC causes sequestration and stabilization of the constitutively internalized ErbB3 in nondegradative endosomal compartments.

The effect of the pan PKC siRNA confirmed that PMA-induced ErbB3 stabilization is PKC mediated. PMA activates both conventional and novel PKCs. The findings that Ro 31-8220, but not Gö 6976, blocked stabilization of ErbB3 may appear conflicting as both are reported to inhibit conventional PKCs. Ro 31-8220 is, however, not specific (Davies, Reddy et al. 2000, Goekjian and Jirousek 2001). Our results are thus in line with previous data showing that the broad spectrum PKC inhibitor rottlerin, but not Gö 6976, inhibits endosomal sequestration of EGFR (Llado, Timpson et al. 2008), and PMA-induced phosphorylation of ErbB2 and ErbB3 (Emkey and Kahn 1997). This strongly suggests that the conventional PKCs are not responsible for the observed effects. The only significant effect of isoform specific siRNAs was upon knockdown of PKC $\delta$. This was a bit surprising since PKC $\delta$ is not reported to be inhibited by Ro 31-8220, and we found no effect of Ro 31-8220 on PMA-induced turnover of PKC $\delta$. The data are, however, in line with previous studies showing that $\mathrm{PKC} \delta$ regulates endosomal recycling of EGFR and ErbB2 (Llado, Timpson et al. 2008, Bailey, Luan et al. 2014). The Ro 31-8220 results may still indicate that also other PKC isoforms are involved. Contrary to other PKCs, PKC $\eta$ has been found to be upregulated upon treatment with PMA. The upregulation is depending on PKCE (which is inhibited by Ro 31-8220) (Pal, Outram et al. 2012). We found no obvious PMA-induced upregulation of PKC $\eta$, but contrary to PKC $\alpha$ and $-\delta, P K C \eta$ was not degraded (data not shown). We thus also knocked down PKC $\delta$ and $-\eta$ in combination, but observed no additional effect compared to knockdown of $\mathrm{PKC} \delta$ alone (data not shown). Altogether, we conclude that $\mathrm{PKC} \delta$ is essential for PMA-induced PKC-dependent stabilization of ErbB3, but cannot exclude that other non-identified PKC isoforms are also involved.

Judged by the level of ErbB3 at the plasma membrane, PMA induced the same initial effect as CHX. This could indicate that activation of PKC inhibits ErbB3 synthesis, but the PMA-induced stabilization of total ErbB3 makes this unlikely. Likewise, since the combination of PMA and CHX had an additive effect on ErbB3 plasma membrane expression, it is likely that they affect ErbB3 differently, and that the effect of PMA is, as shown for EGFR and ErbB2, on internalization and/or recycling of ErbB3.

The monensin-induced down-regulation of ErbB3 from the plasma membrane demonstrates that relatively large amounts of ErbB3 under normal circumstances are constitutively internalized and recycled. The findings that PMA at early time points, up to two hours, showed the same effect as monensin, and that a combination of PMA and monensin within this time frame had no additive effect, further suggest that PMA does not directly induce ErbB3 internalization but rather inhibits recycling of the constitutively internalized ErbB3. At later time points PMA prevented the sustained down-regulation and degradation induced by monensin. The finding that ErbB3 gets degraded upon incubation with monensin alone is at first surprising since monensin inhibits acidification of endocytic compartments (Mollenhauer, Morre et al. 1990). This is, however, in line with previous studies showing that constitutive degradation of ErbB3 can occur in the 
presence of lysosomal inhibitors like chloroquine (Qiu and Goldberg 2002, Fry, Simion et al. 2011, Fosdahl, Dietrich et al. 2017). The ErbB3 stabilization induced by PMA in combination with monensin does thus show that PMA-induced PKC activation not only inhibits recycling but also sorts internalized ErbB3 away from a degradative pathway to a non-degradative endocytic compartment.

The exact nature of this non-degradative endocytic compartment is not clear. In control cells internalized ErbB3 localized to EEA1 or Lamp1 positive compartments, indicating transport along a classic degradative pathway. However, in PMA-treated cells ErbB3 localization to EEA1 and Lamp1 positive compartments was strongly reduced. The ErbB3 positive compartments did not show the perinuclear localization described for the PKC-induced pericentrion (Becker and Hannun 2003). Neither did we observe co-sequestration of ErbB3 along with internalized transferrin. The lack of the perinuclear localization is not surprising since previous studies have shown that ceramide synthesis inhibits formation of the pericentrion in MCF-7 cells (Becker, Kitatani et al. 2005). It should, however, be noted that we upon the use of a ceramide inhibitor (Kitatani, Idkowiak-Baldys et al. 2007) did not detect any pericentrion-like ErbB3 positive structures (data not shown).

One possible reason for the altered sorting is induction of alternative sorting signals. Another possibility is lack of signals for, and/or inhibited sorting to late endosomes and lysosomes. Ubiquitination is known to function as signal for sorting of receptors like the EGFR to late endosomes and lysosomes (Haglund and Dikic 2012). Ubiquitination is also reported to mediate degradation of ErbB3 (Cao, Wu et al. 2007, Fosdahl, Dietrich et al. 2017). Since PMA did not induce ErbB3 ubiquitination, this might represent the lack of signal needed for degradative sorting. In support of this, ErbB3 was ubiquitinated and degraded when PMA was combined with HRG. This suggests that ligand-induced ubiquitination is a signal superior to the possible PKCinduced signal. This is in line with that PMA did not prevent 17-AAG induced degradation of ErbB2 (Bailey, Luan et al. 2014, Dietrich, Malik et al. 2018). Contrary to a previous study (Emkey and Kahn 1997), we did not observe any PMA-induced tyrosine phosphorylation of ErbB3. Since PKCs are serine/threonine kinases, phosphorylation on such residues are more likely. Several studies have shown that activation of PKC causes EGFR Thr-654 phosphorylation, and ErbB2 Thr-686 phosphorylation has also been reported (Ouyang, Gulliford et al. 1996, Ouyang, Gulliford et al. 1998). In ErbB3 the corresponding amino acid is alanine (Ouyang, Gulliford et al. 1998) and whether PKC activation causes phosphorylation of threonine and/or serine residues in ErbB3 is not known. We could not detect any serine/threonine phosphorylation after immunoprecipitating ErbB3 and blotting with anti-phosphoserine/threonine antibodies (data not shown), this may, however, be a matter of antibody sensitivity. The PKC-induced signal(s) causing re-localization and stabilization of ErbB3 do thus remain to be identified. Since PMAinduced activation of $\mathrm{PKC}$ has been suggested to increase endocytosis in general by modulation of early endosomes, and to cause sequestration of a number of receptors, including the constitutively recycling transferrin receptor (Aballay, Stahl et al. 1999, Idkowiak-Baldys, Baldys et al. 2009), the effect can be general and not caused by a signal directly associated with ErbB3. The findings that not only PKC activation, but also PKC inhibition, had effects on ErbB3 expression and degradation do, however, suggest that PKC activity directly controls ErbB3 and regulates to what extent internalized ErbB3 is recycled or sorted for degradation. While a basal PKC activity appears necessary to recycle and keep ErbB3 expression at a constant level, 
increased PKC activity alters the endosomal sorting of ErbB3 and sequester the receptor in specialized non-degradative compartments.

The physiological role of PKC regulated trafficking of receptors like ErbB3 can only be speculated but since PKCs can be activated by several pathways one possible role is to control plasma membrane expression and thus ligand-induced activation of receptors. Increased activation of PKCs will, in a ligand-independent way, sequester receptors inside the cells where ligands have no access. Such a sequestration inhibits ligand-induced activation, without causing degradation of the receptors. Release of receptors from this recycling compartment will allow a rapid reestablishment of receptors at the plasma membrane without the need of new synthesis of receptors.

Acknowledgements: The work was supported by the South-Eastern Norway Regional Health Authority (M. Dietrich, Grant \#2013019), the Norwegian Cancer Society (V. Bertelsen, Grant \#4474487), UNIFOR, Anders Jahre's Foundation for the Promotion of Sciences, and the Olav Raagholt and Gerd Meidel Raagholt Research Foundation.

Conflict of interest: The authors declare that they have no conflicts of interest with the contents of this article. 


\section{References}

Aballay, A., P. D. Stahl and L. S. Mayorga (1999). "Phorbol ester promotes endocytosis by activating a factor involved in endosome fusion." J Cell Sci 112 ( Pt 15): 2549-2557.

Arteaga, C. L. and J. A. Engelman (2014). "ERBB receptors: from oncogene discovery to basic science to mechanism-based cancer therapeutics." Cancer Cell 25(3): 282-303.

Bailey, T. A., H. Luan, E. Tom, T. A. Bielecki, B. Mohapatra, G. Ahmad, M. George, D. L. Kelly, A. Natarajan, S. M. Raja, V. Band and H. Band (2014). "A kinase inhibitor screen reveals protein kinase Cdependent endocytic recycling of ErbB2 in breast cancer cells." J Biol Chem 289(44): 30443-30458. Bao, J., I. Alroy, H. Waterman, E. D. Schejter, C. Brodie, J. Gruenberg and Y. Yarden (2000). "Threonine phosphorylation diverts internalized epidermal growth factor receptors from a degradative pathway to the recycling endosome." J Biol Chem 275(34): 26178-26186.

Becker, K. P. and Y. A. Hannun (2003). "cPKC-dependent sequestration of membrane-recycling components in a subset of recycling endosomes." J Biol Chem 278(52): 52747-52754.

Becker, K. P., K. Kitatani, J. Idkowiak-Baldys, J. Bielawski and Y. A. Hannun (2005). "Selective inhibition of juxtanuclear translocation of protein kinase $\mathrm{C}$ betaII by a negative feedback mechanism involving ceramide formed from the salvage pathway." J Biol Chem 280(4): 2606-2612.

Bertelsen, V., M. M. Sak, K. Breen, M. S. Rødland, L. E. Johannessen, L. M. Traub, E. Stang and I. H. Madshus (2011). "A Chimeric Pre-ubiquitinated EGF Receptor is Constitutively Endocytosed in a ClathrinDependent, but Kinase-Independent Manner." Traffic 12(4): 507-520.

Cao, Z., X. Wu, L. Yen, C. Sweeney and K. L. Carraway, 3rd (2007). "Neuregulin-induced ErbB3

downregulation is mediated by a protein stability cascade involving the E3 ubiquitin ligase Nrdp1." Mol Cell Biol 27(6): 2180-2188.

Davies, S. P., H. Reddy, M. Caivano and P. Cohen (2000). "Specificity and mechanism of action of some commonly used protein kinase inhibitors." Biochem J 351(Pt 1): 95-105.

Dietrich, M., M. S. Malik, F. Nikolaysen, M. Skeie and E. Stang (2018). "Protein kinase C mediated internalization of ErbB2 is independent of clathrin, ubiquitination and Hsp90 dissociation." Exp Cell Res 371(1): 139-150.

Emkey, R. and C. R. Kahn (1997). "Cross-talk between phorbol ester-mediated signaling and tyrosine kinase proto-oncogenes. I. Activation of protein kinase $\mathrm{C}$ stimulates tyrosine phosphorylation and activation of ErbB2 and ErbB3." J Biol Chem 272(49): 31172-31181.

Fosdahl, A. M., M. Dietrich, K. O. Schink, M. S. Malik, M. Skeie, V. Bertelsen and E. Stang (2017). "ErbB3 interacts with Hrs and is sorted to lysosomes for degradation." Biochim Biophys Acta Mol Cell Res 1864(12): 2241-2252.

Fry, W. H., C. Simion, C. Sweeney and K. L. Carraway, 3rd (2011). "Quantity control of the ErbB3 receptor tyrosine kinase at the endoplasmic reticulum." Mol Cell Biol 31(14): 3009-3018.

Goekjian, P. G. and M. R. Jirousek (2001). "Protein kinase C inhibitors as novel anticancer drugs." Expert Opin Investig Drugs 10(12): 2117-2140.

Haglund, K. and I. Dikic (2012). "The role of ubiquitylation in receptor endocytosis and endosomal sorting." J Cell Sci 125(Pt 2): 265-275.

Hunter, T., N. Ling and J. A. Cooper (1984). "Protein kinase C phosphorylation of the EGF receptor at a threonine residue close to the cytoplasmic face of the plasma membrane." Nature 311(5985): 480-483.

Idkowiak-Baldys, J., A. Baldys, J. R. Raymond and Y. A. Hannun (2009). "Sustained receptor stimulation leads to sequestration of recycling endosomes in a classical protein kinase $\mathrm{C}$ - and phospholipase Ddependent manner." J Biol Chem 284(33): 22322-22331.

Kitatani, K., J. Idkowiak-Baldys and Y. A. Hannun (2007). "Mechanism of inhibition of sequestration of protein kinase $\mathrm{C}$ alpha/betaII by ceramide. Roles of ceramide-activated protein phosphatases and phosphorylation/dephosphorylation of protein kinase C alpha/betaII on threonine 638/641." J Biol Chem 282(28): 20647-20656.

Liu, M., J. Idkowiak-Baldys, P. L. Roddy, A. Baldys, J. Raymond, C. J. Clarke and Y. A. Hannun (2013). "Sustained activation of protein kinase $C$ induces delayed phosphorylation and regulates the fate of epidermal growth factor receptor." PLoS One 8(11): e80721.

Llado, A., P. Timpson, S. Vila de Muga, J. Moreto, A. Pol, T. Grewal, R. J. Daly, C. Enrich and F. Tebar (2008). "Protein kinase Cdelta and calmodulin regulate epidermal growth factor receptor recycling from early endosomes through Arp2/3 complex and cortactin." Mol Biol Cell 19(1): 17-29. 
Lund, K. A., C. S. Lazar, W. S. Chen, B. J. Walsh, J. B. Welsh, J. J. Herbst, G. M. Walton, M. G.

Rosenfeld, G. N. Gill and H. S. Wiley (1990). "Phosphorylation of the epidermal growth factor receptor at threonine 654 inhibits ligand-induced internalization and down-regulation." J Biol Chem 265(33): $20517-$ 20523.

Mollenhauer, H. H., D. J. Morre and L. D. Rowe (1990). "Alteration of intracellular traffic by monensin; mechanism, specificity and relationship to toxicity." Biochim Biophys Acta 1031(2): 225-246.

Mosior, M. and A. C. Newton (1996). "Calcium-independent binding to interfacial phorbol esters causes protein kinase $\mathrm{C}$ to associate with membranes in the absence of acidic lipids." Biochemistry 35(5): 16121623.

Nixon, J. S., J. Bishop, D. Bradshaw, P. D. Davis, C. H. Hill, L. H. Elliott, H. Kumar, G. Lawton, E. J. Lewis, M. Mulqueen and et al. (1992). "The design and biological properties of potent and selective inhibitors of protein kinase C." Biochem Soc Trans 20(2): 419-425.

Ouyang, X., T. Gulliford and R. J. Epstein (1998). "The duration of phorbol-inducible ErbB2 tyrosine dephosphorylation parallels that of receptor endocytosis rather than threonine-686 phosphorylation: implications for the physiological role of protein kinase $\mathrm{C}$ in growth factor receptor signalling."

Carcinogenesis 19(11): 2013-2019.

Ouyang, X., T. Gulliford, H. Zhang, G. C. Huang and R. Epstein (1996). "Human cancer cells exhibit protein kinase C-dependent c-erbB-2 transmodulation that correlates with phosphatase sensitivity and kinase activity." J Biol Chem 271(36): 21786-21792.

Pal, D., S. P. Outram and A. Basu (2012). "Novel regulation of protein kinase C-eta." Biochem Biophys Res Commun 425(4): 836-841.

Parker, P. J., L. Bosca, L. Dekker, N. T. Goode, N. Hajibagheri and G. Hansra (1995). "Protein kinase C (PKC)-induced PKC degradation: a model for down-regulation." Biochem Soc Trans 23(1): 153-155.

Pinkas-Kramarski, R., M. Shelly, S. Glathe, B. J. Ratzkin and Y. Yarden (1996). "Neu Differentiation Factor/Neuregulin Isoforms Activate Distinct Receptor Combinations." Journal of Biological Chemistry 271(32): 19029-19032.

Qiu, X. B. and A. L. Goldberg (2002). "Nrdp1/FLRF is a ubiquitin ligase promoting ubiquitination and degradation of the epidermal growth factor receptor family member, ErbB3." Proc Natl Acad Sci U S A 99(23): 14843-14848.

Roskoski, R., Jr. (2014). "The ErbB/HER family of protein-tyrosine kinases and cancer." $\underline{\text { Pharmacol Res }}$ 79: 34-74.

Rozengurt, E. (2007). "Mitogenic signaling pathways induced by G protein-coupled receptors." $\underline{\mathrm{J} \text { Cell }}$ Physiol 213(3): 589-602.

Sak, M. M., K. Breen, S. B. Ronning, N. M. Pedersen, V. Bertelsen, E. Stang and I. H. Madshus (2012).

"The oncoprotein ErbB3 is endocytosed in the absence of added ligand in a clathrin-dependent manner." Carcinogenesis 33(5): 1031-1039.

Sorkin, A. and L. K. Goh (2009). "Endocytosis and intracellular trafficking of ErbBs." Exp Cell Res 315(4): 683-696.

Szymanska, M., A. M. Fosdahl, C. Raiborg, M. Dietrich, K. Liestøl, E. Stang and V. Bertelsen (2016).

"Interaction with epsin 1 regulates the constitutive clathrin-dependent internalization of ErbB3."

Biochimica et Biophysica Acta (BBA) - Molecular Cell Research 1863(6, Part A): 1179-1188.

Vuong, T. T., C. Berger, V. Bertelsen, M. S. Rødland, E. Stang and I. H. Madshus (2013).

"Preubiquitinated chimeric ErbB2 is constitutively endocytosed and subsequently degraded in lysosomes." Experimental Cell Research 319(3): 32-45.

Warren, C. M., K. Kani and R. Landgraf (2006). "The N-terminal domains of neuregulin 1 confer signal attenuation." J Biol Chem 281(37): 27306-27316.

Waterman, H., I. Sabanai, B. Geiger and Y. Yarden (1998). "Alternative intracellular routing of ErbB receptors may determine signaling potency." J Biol Chem 273(22): 13819-13827.

Wilkinson, S. E., P. J. Parker and J. S. Nixon (1993). "Isoenzyme specificity of bisindolylmaleimides, selective inhibitors of protein kinase C." Biochem J 294 ( Pt 2): 335-337. 


\section{FIGURE LEGENDS}

FIGURE 1. PKC activation inhibits constitutive ErbB3 degradation (A) MCF-7 cells were treated with CHX $(25 \mu \mathrm{g} / \mathrm{ml})$ and DMSO (vehicle control), or PMA (100nM) alone for indicated times. Cell lysates were subjected to immunoblotting using antibodies to ErbB3 (Ab-6); PKC $\alpha$ and PKC $\delta$ as controls for PKC activation, and tubulin (loading control). One representative experiment out of three is shown. (B) Cells were pre-treated for 15 min with CHX before further incubation with CHX and PMA or DMSO (vehicle control) for the indicated times. Cell lysates were subjected to immunoblotting as in A. One representative experiment out of three is shown. (C) Net luminescence of the bands corresponding to ErbB3 in (B) was quantified, normalized to tubulin and plotted as normalized to the ErbB3 level in cells not exposed to $\mathrm{CHX}$. The quantifications represent data from three independent experiments and are shown as mean \pm SD. n.s. non-significant, $* \mathrm{p}<0.05, * * \mathrm{p}<0.01$.

FIGURE 2. PKC inhibition does not alter ErbB3 stability (A) MCF-7 cells, pre-treated with CHX for $15 \mathrm{~min}$, were further incubated with or without Ro 31-8220 $(1 \mu \mathrm{M})$ for $15 \mathrm{~min}$ before a final incubation with or without Ro 31-8220 \pm PMA for the indicated times. Since PMA and Ro 31-8220 were dissolved using DMSO, DMSO was included along with $\mathrm{CHX}$ as vehicle control. Cell lysates were subjected to immunoblotting using antibodies to ErbB3 (Ab-6); PKC $\alpha$ and $\mathrm{PKC} \delta$ as controls for PKC activation, and tubulin (loading control). One representative experiment out of three is shown. (B) Net luminescence of the bands corresponding to ErbB3 was quantified, normalized to tubulin and plotted as normalized to the ErbB3 level in cells not exposed to CHX. The quantifications represent data from three independent experiments and are shown as mean $\pm \mathrm{SD}$. $* \mathrm{p}<0.05$, ** $\mathrm{p}<0.01, * * * \mathrm{p}<0.001$.

FIGURE 3. PKC regulates the plasma membrane expression of ErbB3 (A) MCF-7 cells were treated with cycloheximide (CHX) $(25 \mu \mathrm{g} / \mathrm{ml})$ or PMA $(100 \mathrm{nM})$ alone or in combination for the indicate times. DMSO was included as vehicle control. Fixed non-permeabilized cells were stained with a PE-conjugated antibody to ErbB3 (1B4C3) and subjected to flow cytometry. Data from 3 independent experiments was normalized to untreated cells and pooled. Mean values $\pm \mathrm{SD}$ were plotted. $* \mathrm{p}<0.05, * * \mathrm{p}<0.01$, *** $\mathrm{p}<0.001$. (B) Cells treated with CHX alone, Ro 31-8220 (1 $\mu \mathrm{M})$ alone, CHX combined with DMSO (vehicle control), CHX combined with PMA, CHX combined with Ro 31-8220, or CHX combined with PMA and Ro 31-8220 were stained with an APC-conjugated antibody to ErbB3 (1B4C3) and analyzed as in A. * $\mathrm{p}<0.05, * * \mathrm{p}<0.01, * * * \mathrm{p}<0.001$.

FIGURE 4. PKC regulates intracellular sorting of ErbB3 (A) MCF-7 cells were incubated with a mouse anti-ErbB3 (1B4C3) antibody for $30 \mathrm{~min}$ at $18^{\circ} \mathrm{C}$, washed and chased with PMA (100 nM) or the corresponding amount of DMSO for $30 \mathrm{~min}$ at $37^{\circ} \mathrm{C}$. Localization of the anti-ErbB3 antibody was detected by staining for mouse IgG. Early endosomes were localized by staining for EEA1. The cells were analyzed by confocal microscopy. Insets show high magnifications of the correspondingly framed areas. Scale bar represents $20 \mu \mathrm{m}$. Micrographs representative for three experiments are shown. $(B)$ Microscopy pictures of cells incubated as in A were analyzed for co-localization as described in materials and methods. Asterisks indicate $\mathrm{P}$ value $<0.0001$.

FIGURE 5. PKC activation inhibits lysosomal sorting of ErbB3 while PKC inhibition does not alter sorting of ErbB3 (A) MCF-7 cells were incubated with a mouse anti-ErbB3 (1B4C3) antibody for 30 
min at $18^{\circ} \mathrm{C}$, washed and chased with PMA $(100 \mathrm{nM})$, or the corresponding amount of DMSO for 30 min at $37^{\circ} \mathrm{C}$. Localization of the anti-ErbB3 antibody was detected by staining for mouse IgG. Late endosomes/lysosomes were localized with an antibody to LAMP1. (B) MCF-7 cells, incubated with mouse anti-ErbB3 antibody for $30 \mathrm{~min}$ at $18^{\circ} \mathrm{C}$ as in A, were chased with Ro 31-8220 $(1 \mu \mathrm{M})$ for $30 \mathrm{~min}$ at $37^{\circ} \mathrm{C}$. Early endosomes were localized by staining for EEA1. The cells were analyzed by confocal microscopy. Insets show high magnifications of the correspondingly framed areas. Scale bar represents 20 $\mu \mathrm{m}$. Micrographs representative for three experiments are shown.

FIGURE 6. Inhibited recycling does not alter PMA-induced stabilization and intracellular sorting of ErbB3 (A-B) MCF-7 cells were treated with CHX $(25 \mu \mathrm{g} / \mathrm{ml})$, the recycling inhibitor Monensin (10 $\mu \mathrm{M})$ and/or PMA (100 $\mathrm{nM}$ ) for indicated times. DMSO was added as vehicle control. (A) Fixed nonpermeabilized cells were stained with a PE-conjugated antibody to ErbB3 (1B4C3) and subjected to flow cytometry. Data from 3 independent experiments was normalized to untreated cells and pooled. Mean values $\pm \mathrm{SD}$ were plotted. $* \mathrm{p}<0.05$, ** $\mathrm{p}<0.01$, *** $\mathrm{p}<0.001$. (B) Cell lysates were subjected to immunoblotting using antibodies to ErbB3 (Ab-6); $\mathrm{PKC} \alpha$ and $\mathrm{PKC} \delta$ (PKC activation controls), and tubulin (loading control). One representative experiment out of three is shown. (C) Net luminescence of the bands corresponding to ErbB3 was quantified, normalized to tubulin and plotted as normalized to the ErbB3 level in cells not exposed to CHX. The quantifications represent data from three independent experiments and are shown as mean \pm SD. $* \mathrm{p}<0.05$. (D) MCF-7 cells were incubated with $10 \mu \mathrm{M}$ Monensin for $120 \mathrm{~min}$ at $37^{\circ} \mathrm{C}$. ErbB3 was localized by staining with an ErbB3 (1B4C3) antibody and late endosomes/lysosomes were localized by staining for Lamp1. The cells were analyzed by confocal microscopy. Inset shows high magnification of the correspondingly framed area. Scale bar represents 20 $\mu \mathrm{m}$. Micrographs representative for three experiments are shown.

FIGURE 7. PMA does not inhibit heregulin-induced down-regulation of ErbB3 MCF-7 cells, preincubated for $15 \mathrm{~min}$ with CHX $(25 \mu \mathrm{g} / \mathrm{ml})$, were further incubated with CHX (DMSO was included as vehicle control), or CHX along with PMA (100 nM); HRG (10 nM), or PMA combined with HRG for indicated times. (A) Fixed non-permeabilized cells were stained with a PE-conjugated antibody to ErbB3 (1B4C3) and subjected to flow cytometry. Data from 3 independent experiments was normalized to untreated cells and pooled. Mean values \pm SD were plotted. $* \mathrm{p}<0.05, * * \mathrm{p}<0.01, * * * \mathrm{p}<0.001$. (B) Cell lysates were subjected to immunoblotting using antibodies to ErbB3 (D22C5); PKC $\alpha$, PKC $\delta$ (PKC activation controls) and tubulin (loading control). One representative experiment out of three is shown. (C) Net luminescence of the bands corresponding to ErbB3 was quantified, normalized to tubulin and plotted as normalized to the ErbB3 level in cells not exposed to CHX. The quantifications represent data from three independent experiments and are shown as mean $\pm \mathrm{SD} . * \mathrm{p}<0.05$.

FIGURE 8. ErbB3 is ubiquitinated and tyrosine phosphorylated upon stimulation with heregulin but not PMA MCF-7 cells were incubated for $20 \mathrm{~min}$ at $37^{\circ} \mathrm{C}$ with PMA (100 nM) and/or 5 min with HRG (10 nM). Cell lysates were subjected to immunoprecipitation under denaturing conditions using mouse anti-ErbB3 (Ab-6) antibody. Non-coated beads were used as control (-Ab). The immunoprecipitated material was analyzed by immunoblotting using antibodies to ubiquitin (Ubi), phosphorylated tyrosine (pTyr) and ErbB3 (Ab-6). One representative experiment out of three is shown. 
FIGURE 9. Gö 6976 does not inhibit PMA-induced stabilization of ErbB3 A) MCF-7 cells, pretreated with CHX for 15 min, were further incubated with or without Gö $6976(3 \mu \mathrm{M})$ for 15 min before a final incubation with or without Gö $6976 \pm$ PMA for the indicated times. Since PMA and Gö 6976 were dissolved using DMSO, DMSO was included along with CHX as vehicle control. Cell lysates were subjected to immunoblotting using antibodies to ErbB3 (D22C5), PKC $\delta$ as control for PKC activation, and tubulin (loading control). One representative experiment out of three is shown. (B) Net luminescence of the bands corresponding to ErbB3 was quantified, normalized to tubulin and plotted as normalized to the ErbB3 level in cells not exposed to CHX. The quantifications represent data from three independent experiments.

FIGURE 10. A general PKC knockdown inhibits PMA-induced stabilization of ErbB3 A) MCF-7 cells, transfected with non-targeting (NT) control siRNA or with pan PKC siRNA, were preincubated with CHX for 15 min before final incubation with DMSO (vehicle control) or PMA for the indicated times. Cell lysates were subjected to immunoblotting using antibodies to ErbB3 (D22C5), PKC $\alpha, \mathrm{PKC} \delta$ and $\mathrm{PKC} \eta$ as controls for PKC knockdown, and tubulin (loading control). One representative experiment out of three is shown. (B) Net luminescence of the bands corresponding to ErbB3 was quantified, normalized to tubulin and plotted as normalized to the ErbB3 level in cells not exposed to CHX. The quantifications represent data from three independent experiments and are shown as mean $\pm \mathrm{SD}$. * $\mathrm{p}<0.05, * * \mathrm{p}<0.01, * * * \mathrm{p}<0.001$.

FIGURE 11. Knockdown PKC $\delta$ inhibits PMA-induced stabilization of ErbB3 A) MCF-7 cells, transfected with non-targeting (NT) control siRNA or with $\mathrm{PKC} \delta$ siRNA, were preincubated with $\mathrm{CHX}$ for 15 min before final incubation with DMSO (vehicle control) or PMA for the indicated times. Cell lysates were subjected to immunoblotting using antibodies to ErbB3 (D22C5), PKC $\delta$, and tubulin (loading control). One representative experiment out of three is shown. (B) Net luminescence of the bands corresponding to ErbB3 was quantified, normalized to tubulin and plotted as normalized to the ErbB3 level in cells not exposed to $\mathrm{CHX}$. The quantifications represent data from three independent experiments and are shown as mean $\pm \mathrm{SD} . * \mathrm{p}<0.05, * * \mathrm{p}<0.01$. 
Figure 1

A

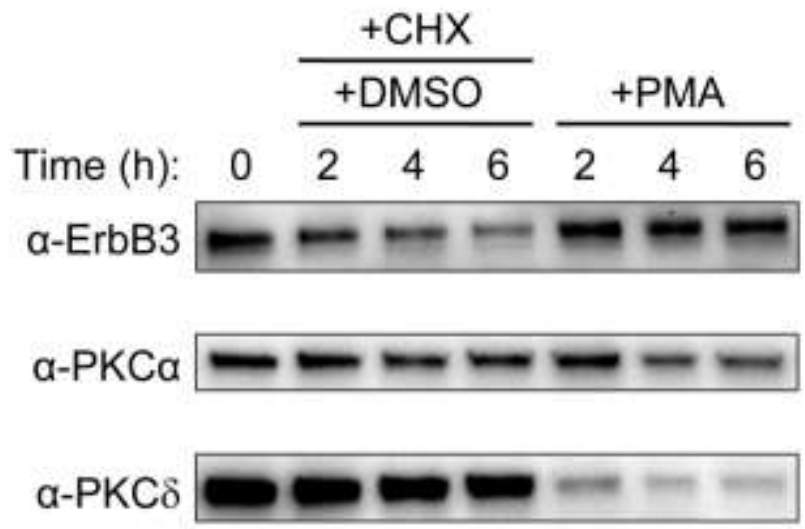

$\alpha-T u b u l i n$

B

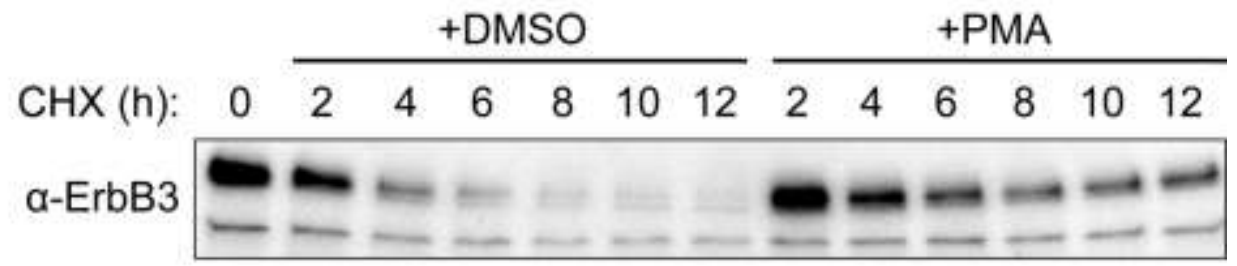

$\alpha-P K C \alpha=-\infty-\infty-\infty-\cdots-\cdots-\cdots$

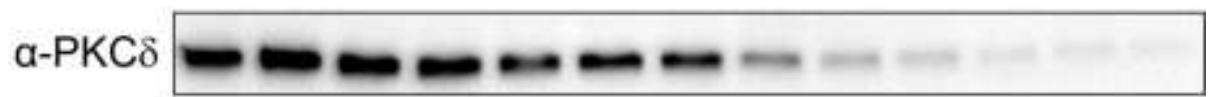

$\alpha-T u b u l i n$

ow

C

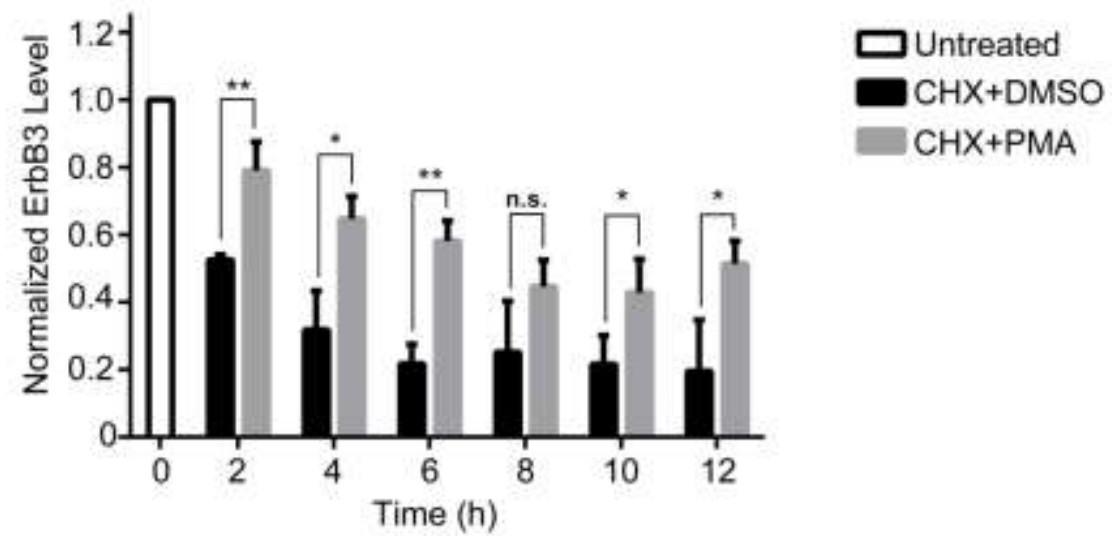


Figure 2

A

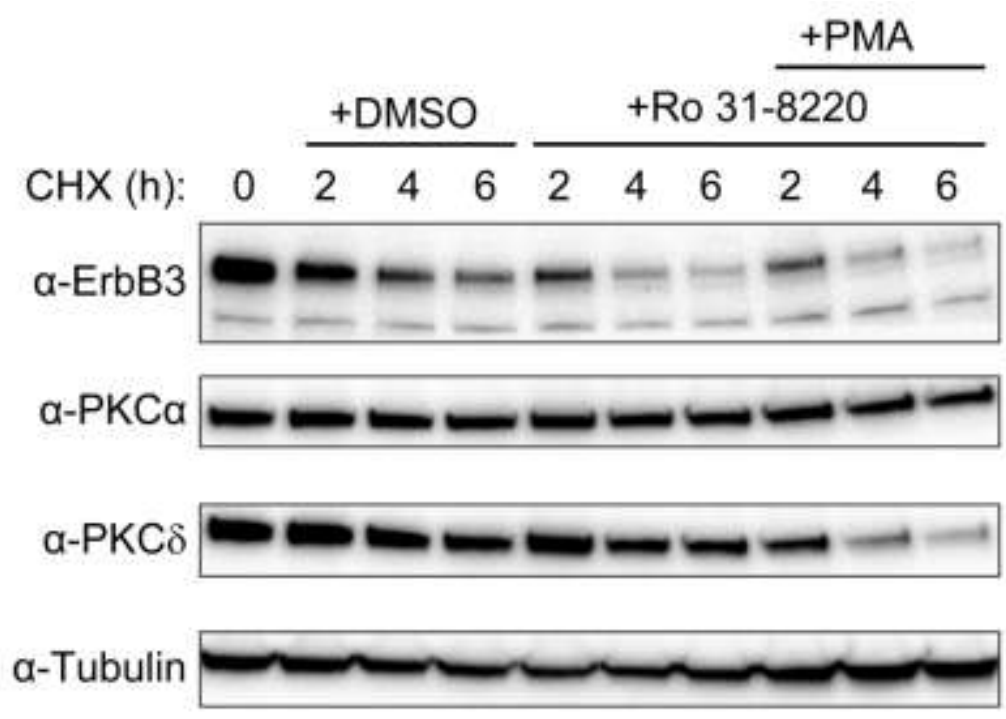

B

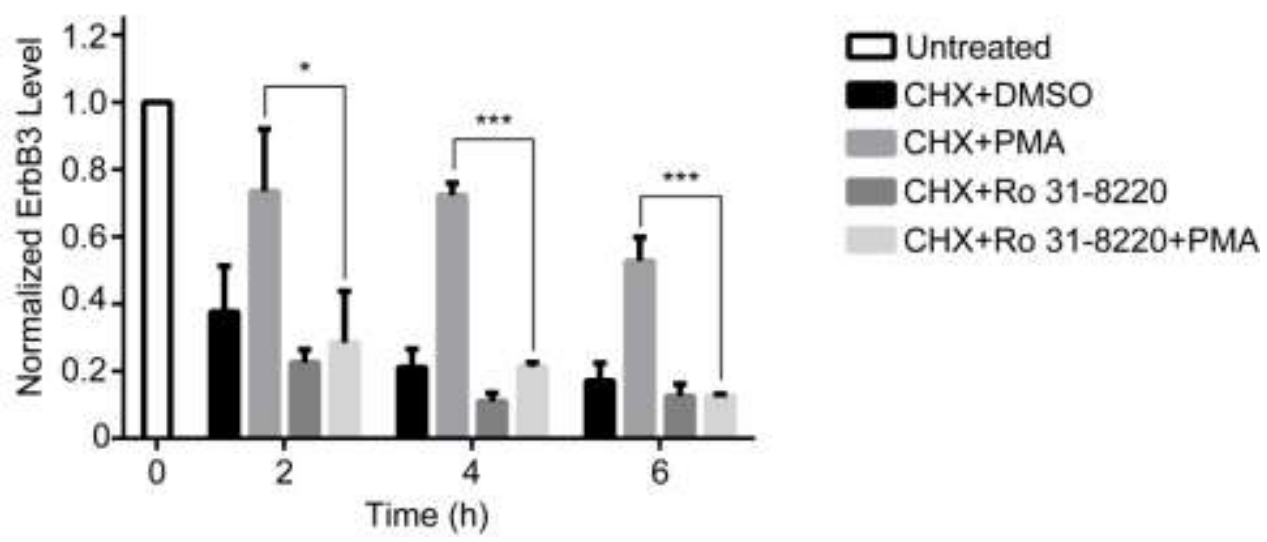


Figure 3

A

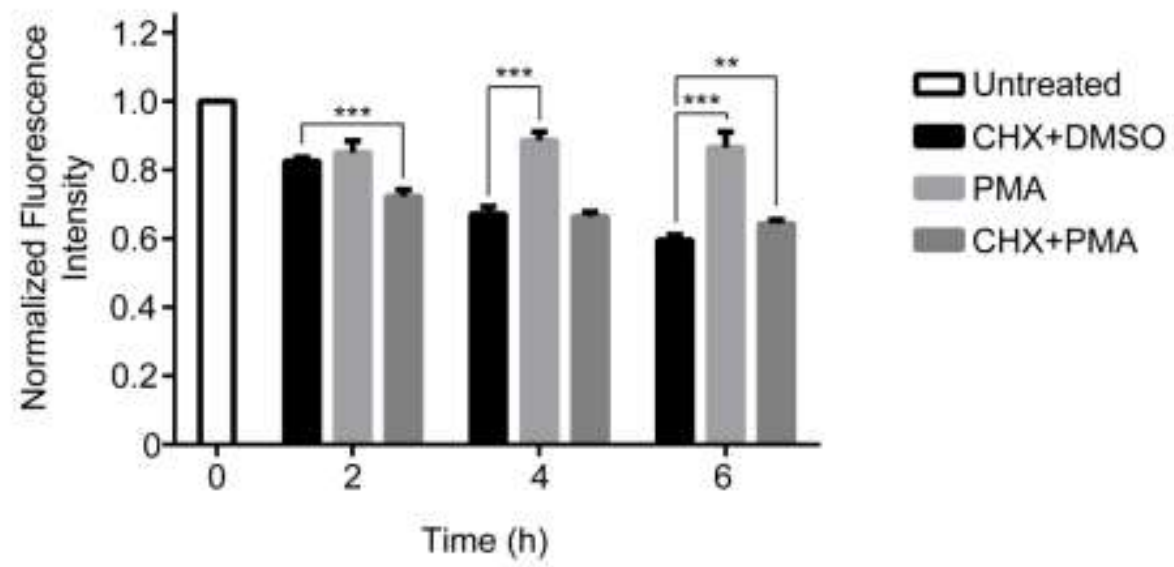

B

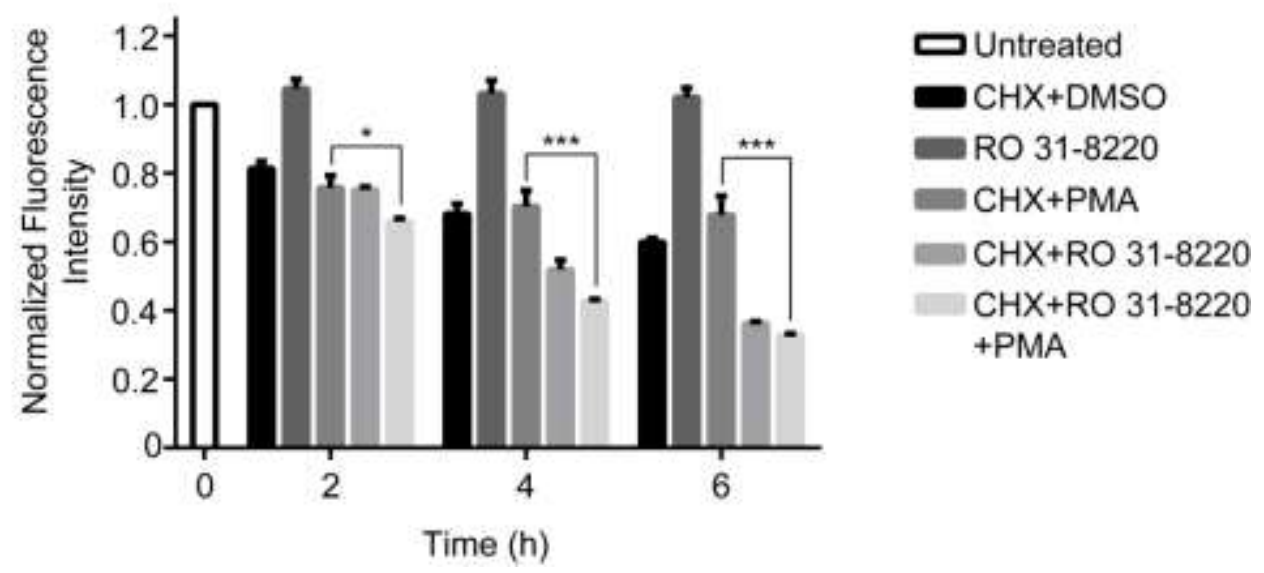


Figure 4

A

$\alpha-$ ErbB3
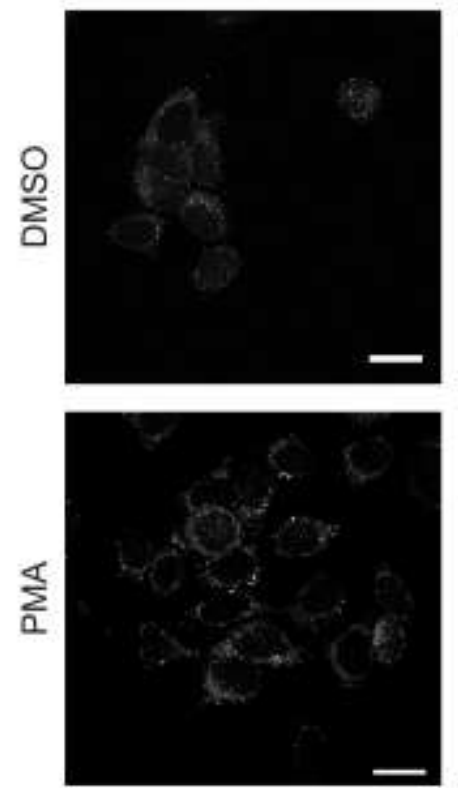

B
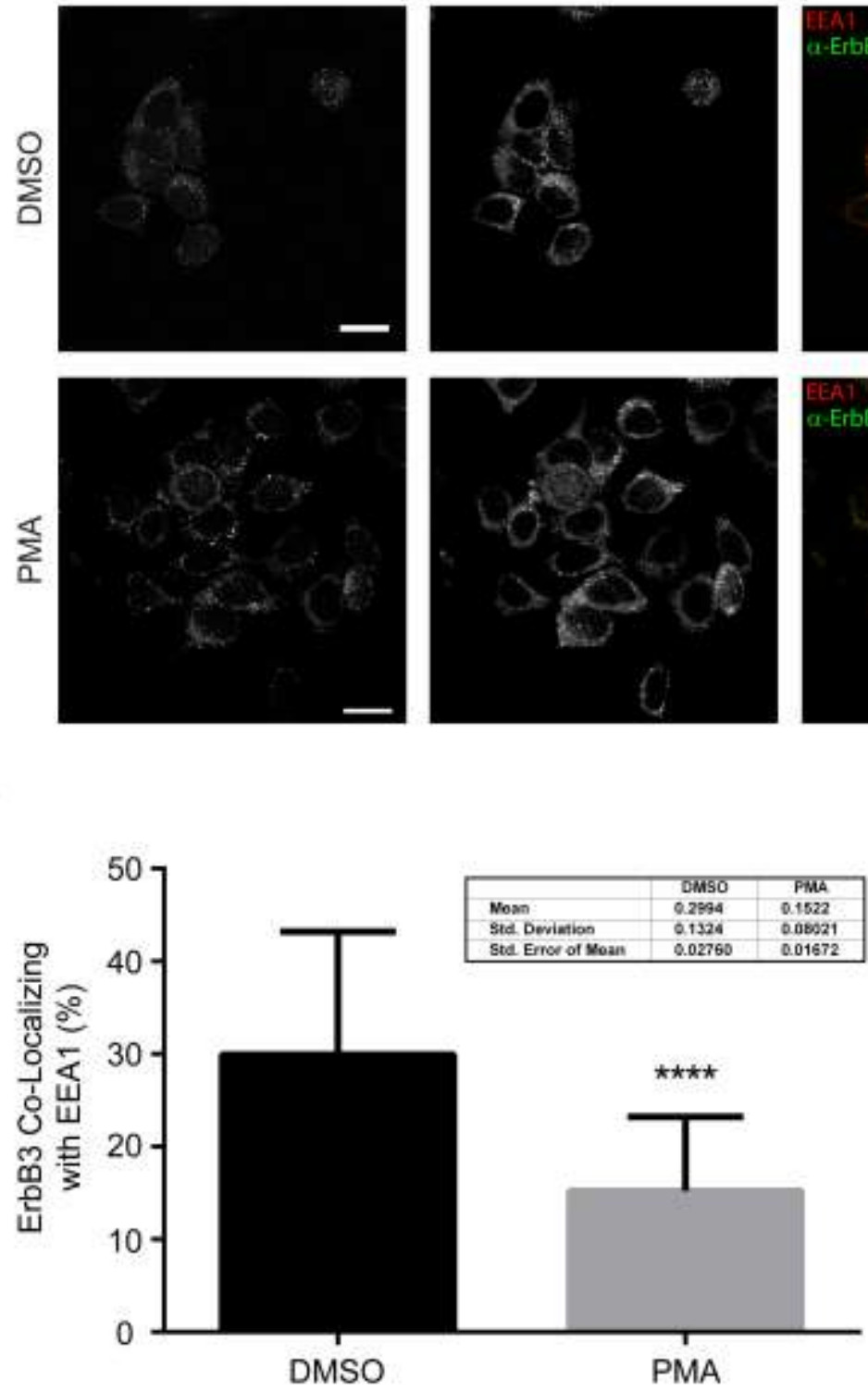

Merge
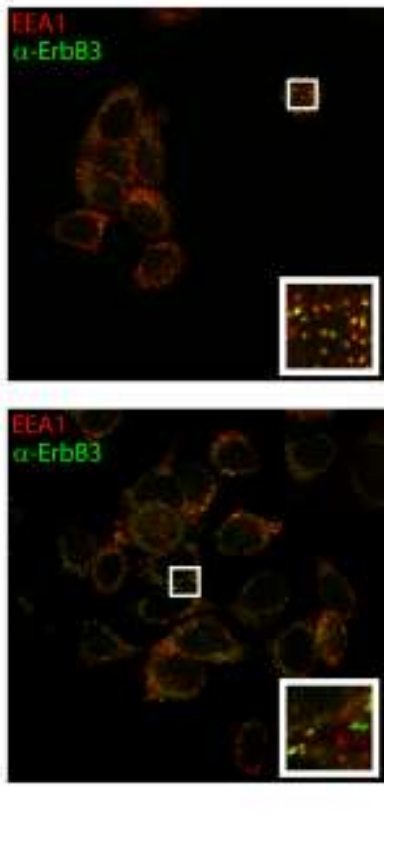

. 
Figure 5

A
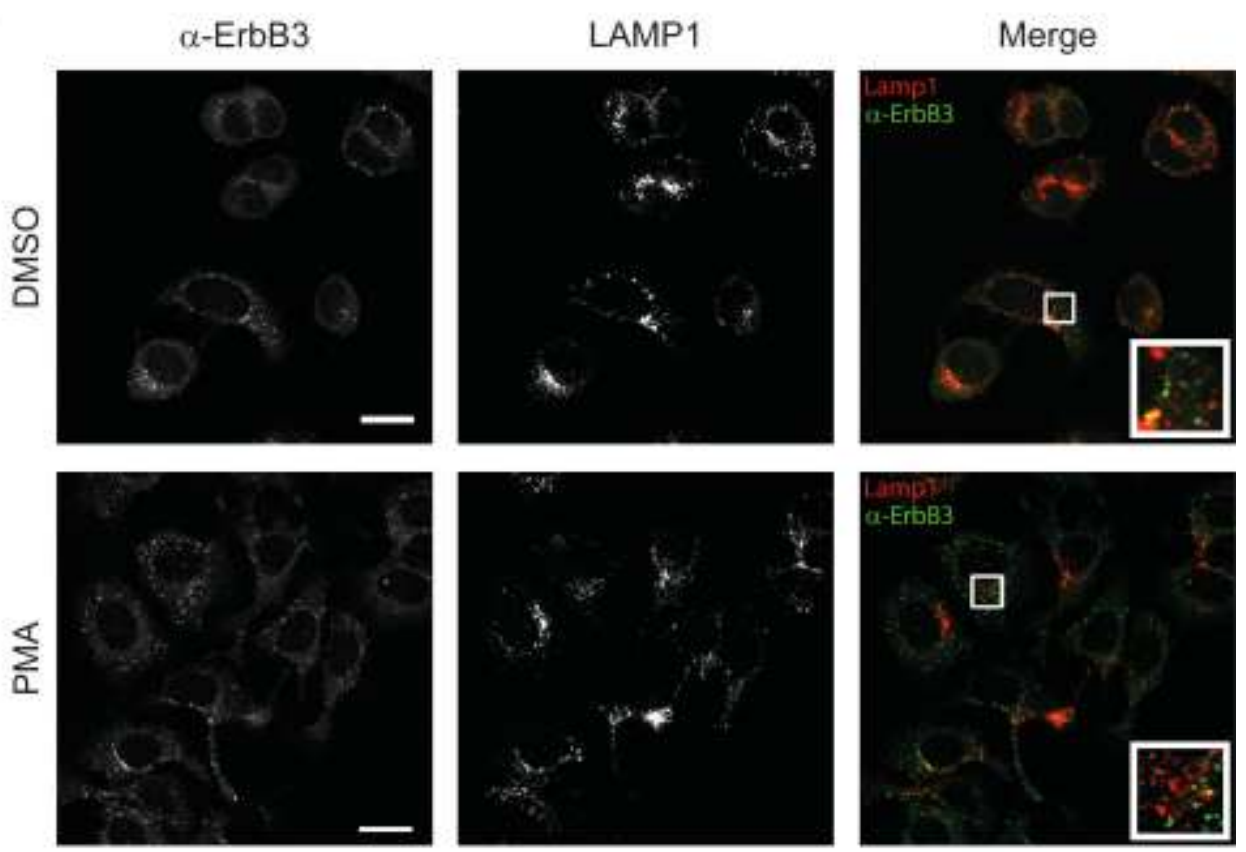

B

$\alpha$-ErbB3

EEA1
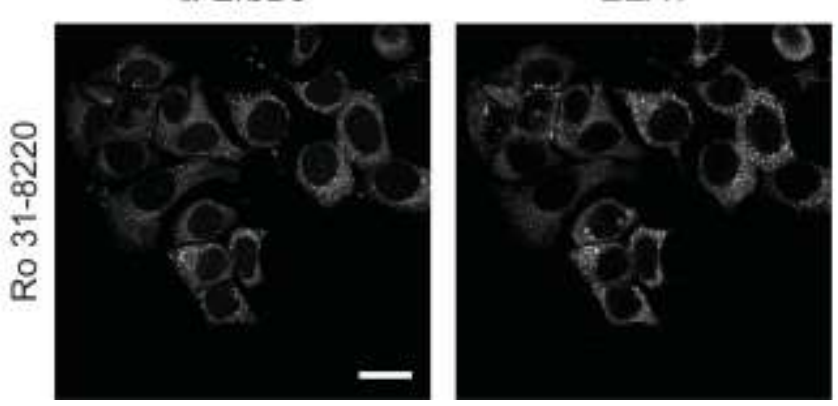

Merge

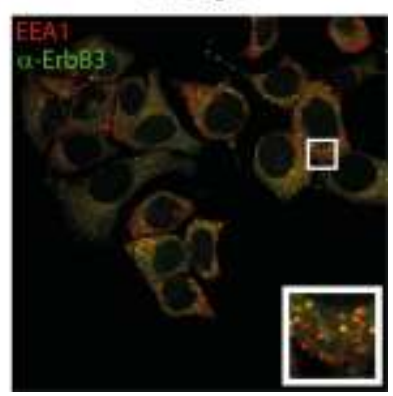


Figure 6

A

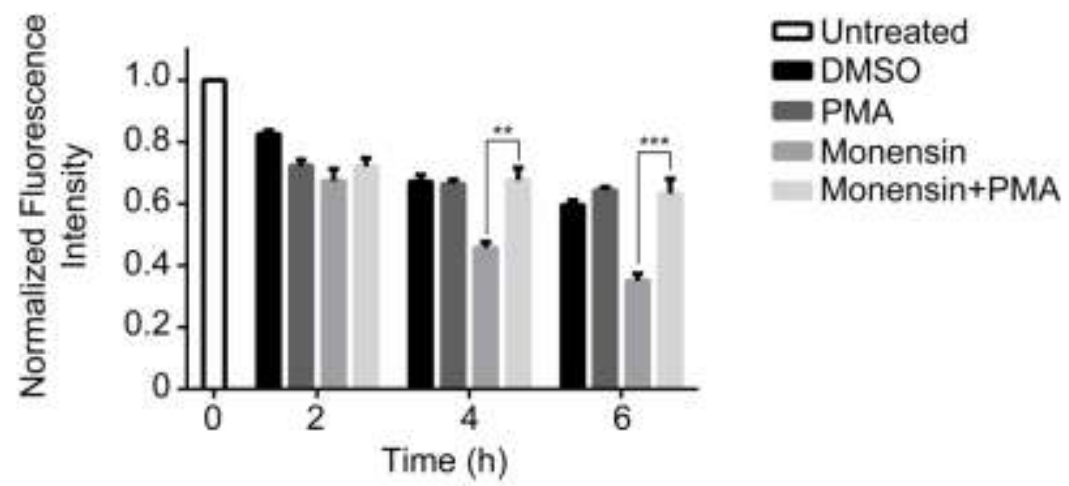

B

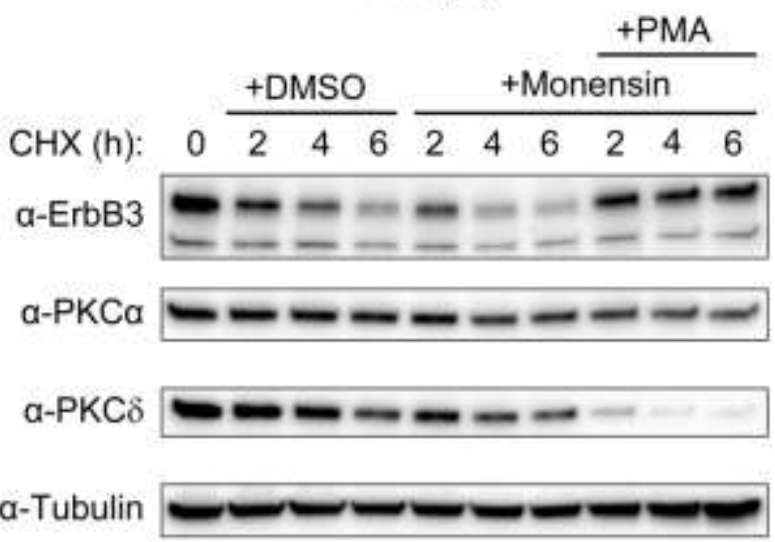

C

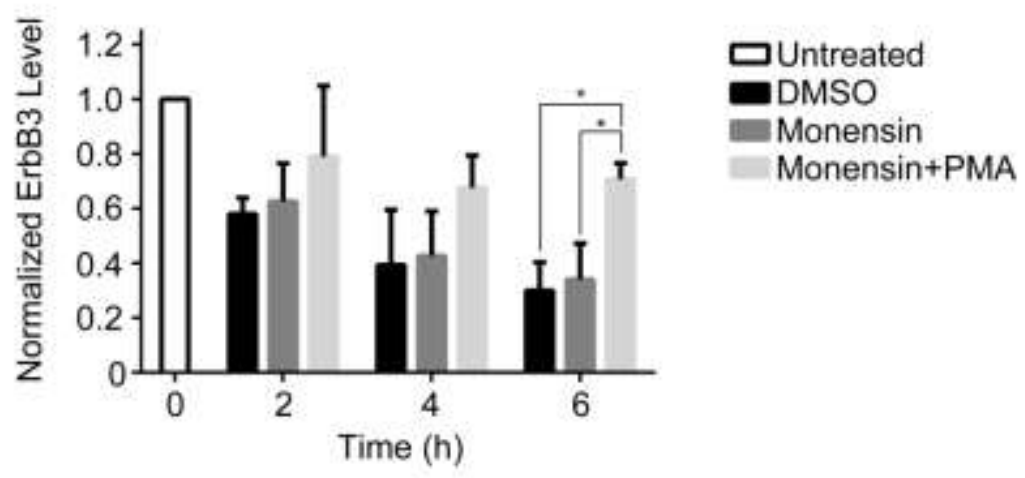

D

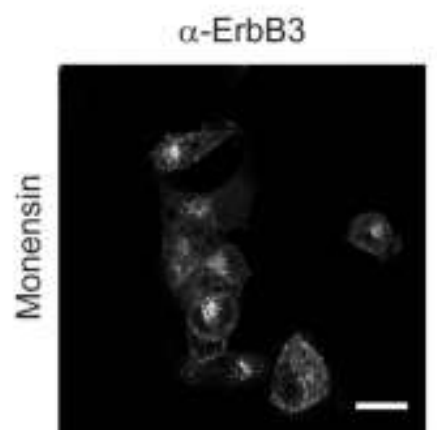

LAMP1

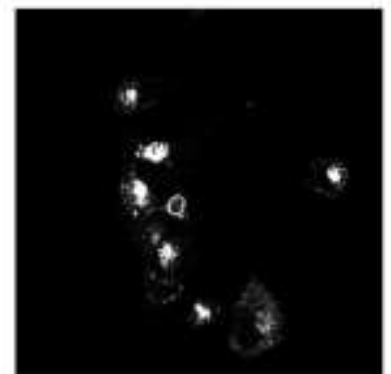

Merge

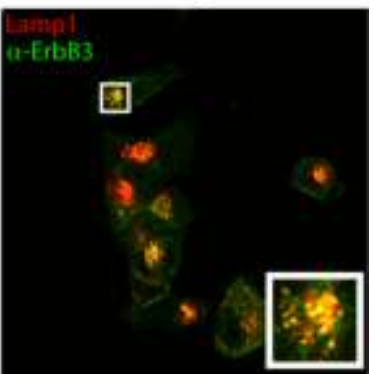


Figure 7

A

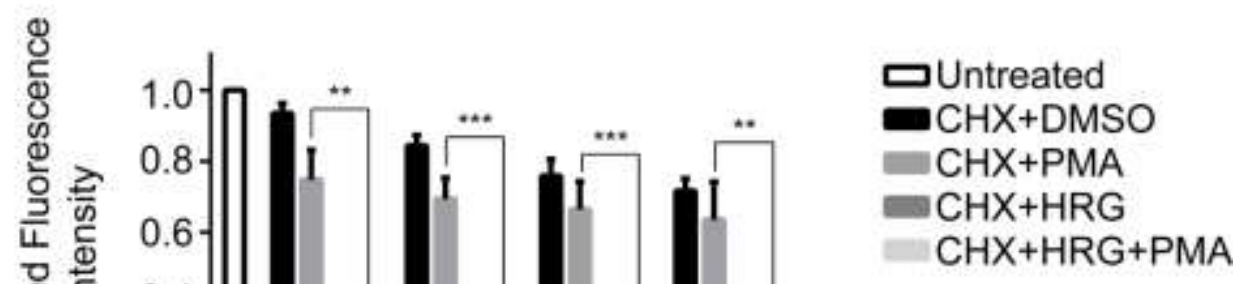

B

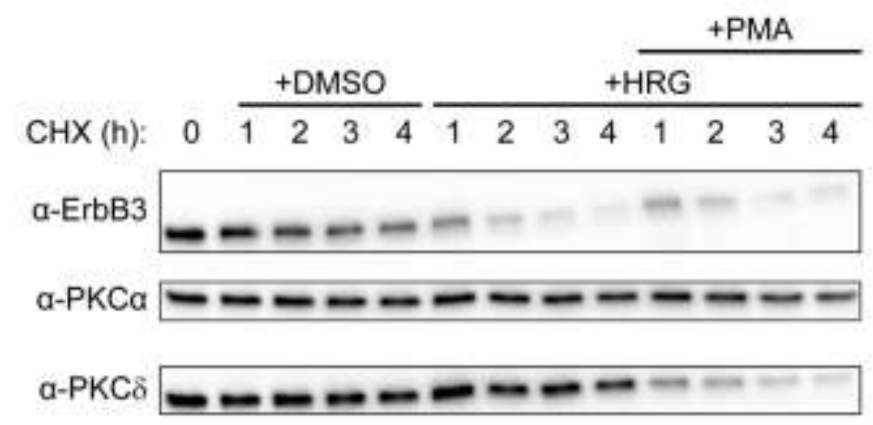

a-Tubulin $-\infty-\infty-\infty-\infty-\infty$

C

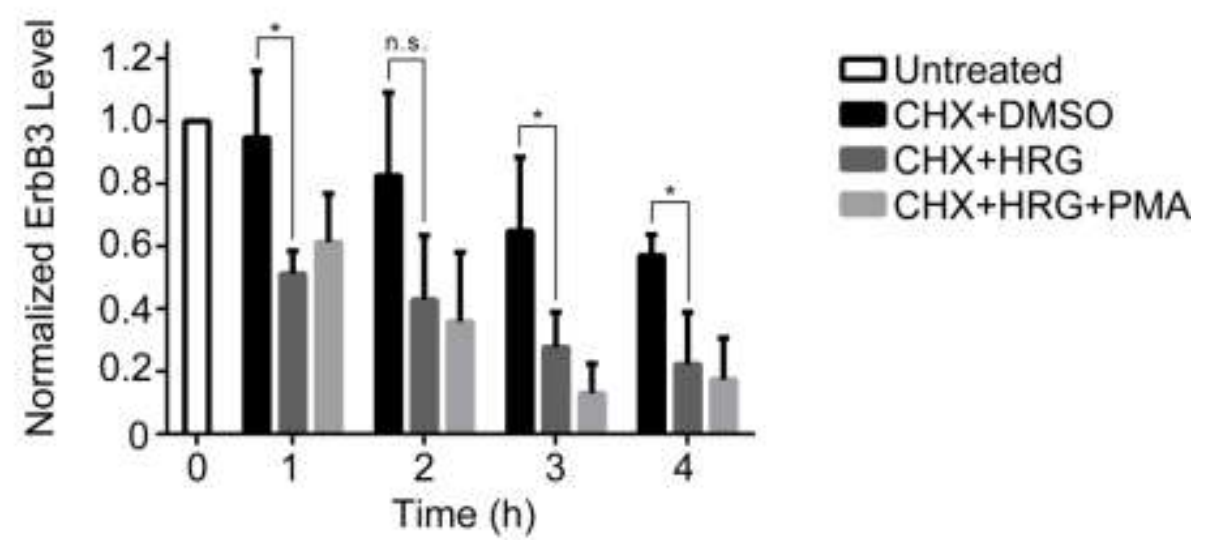


Figure 8

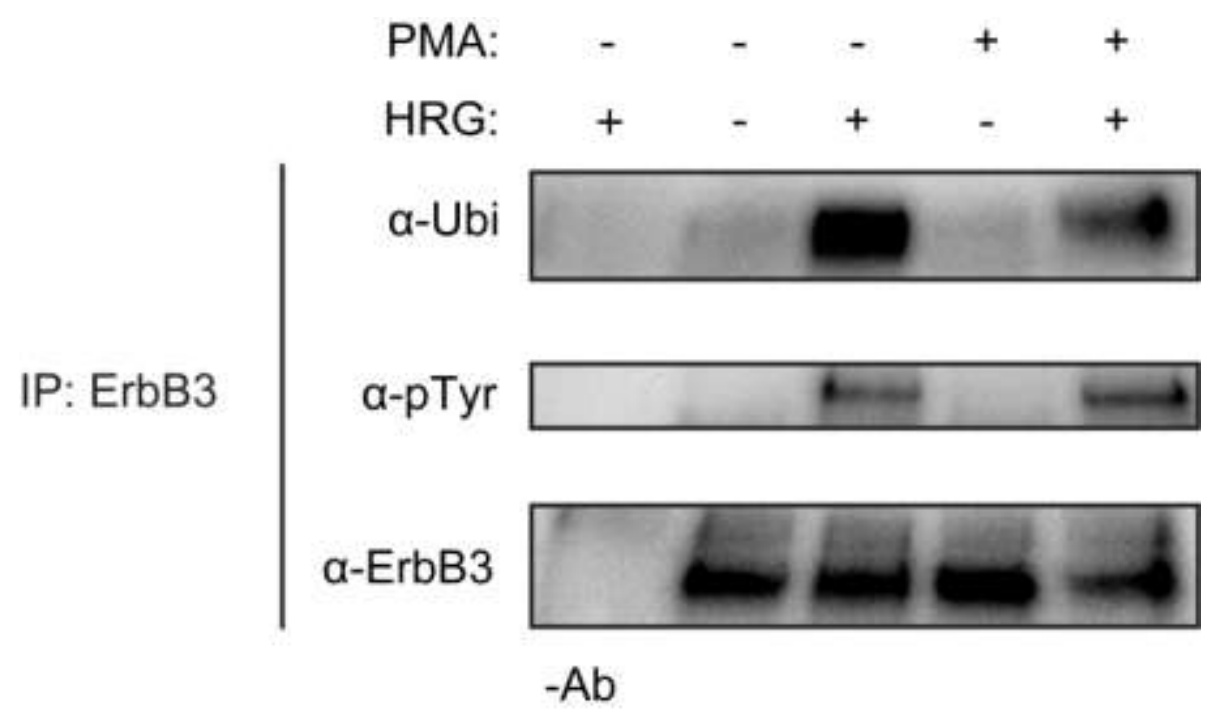


Figure 9

A

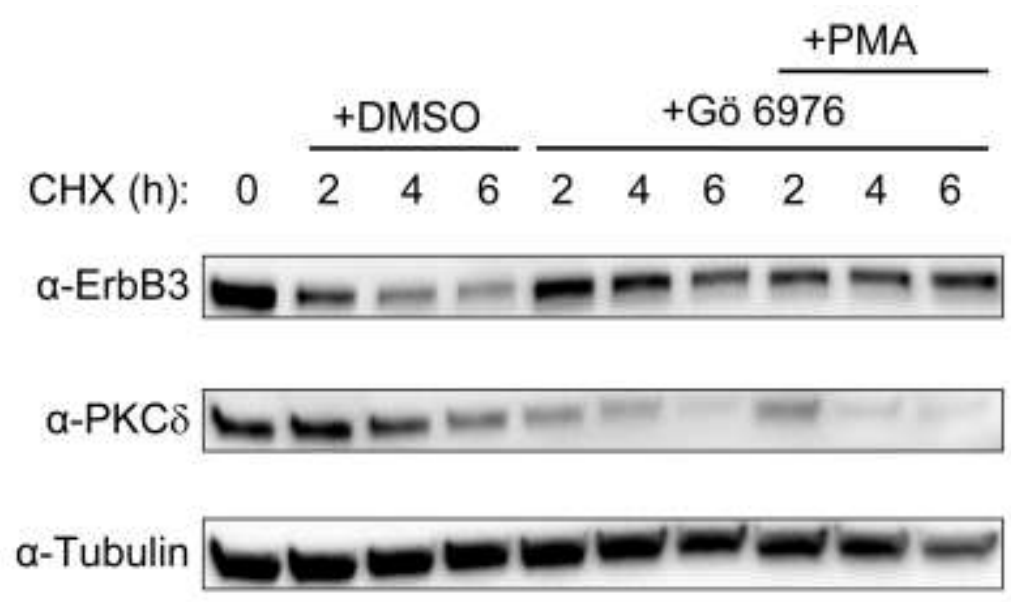

B

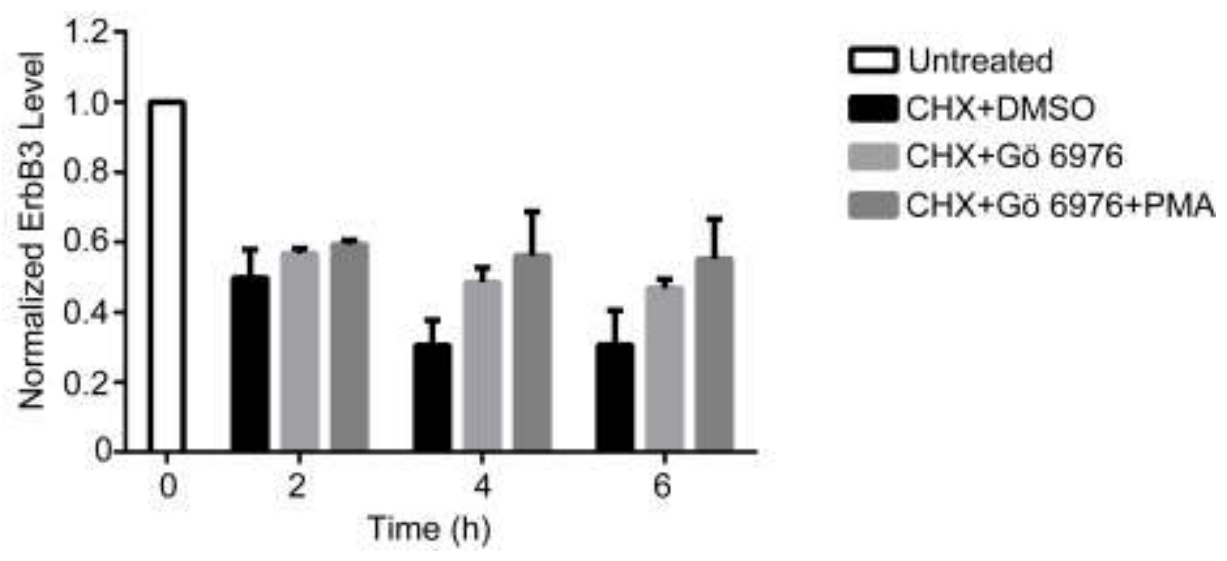


Figure 10

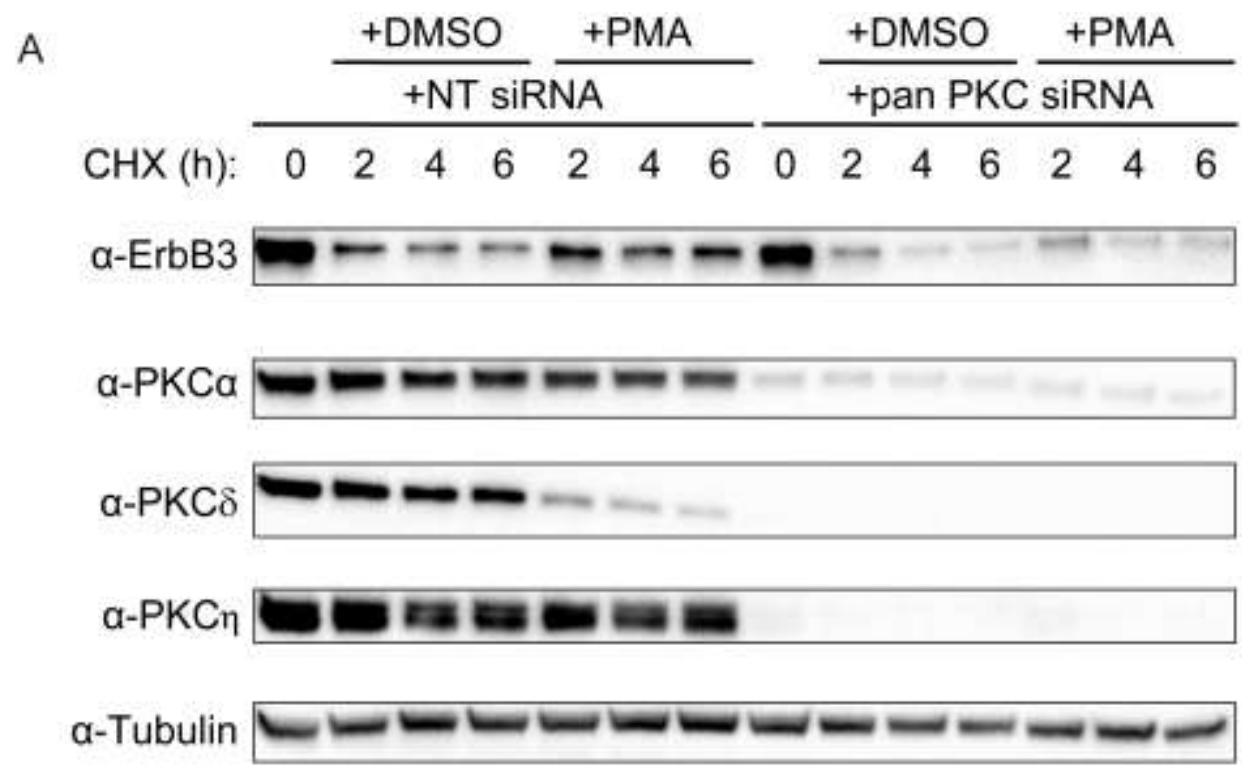

B

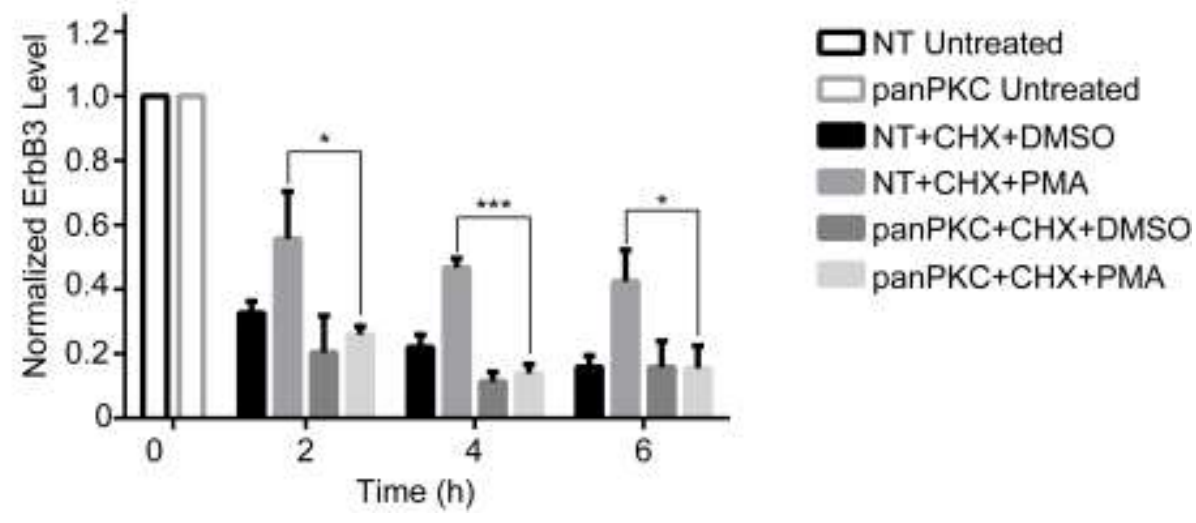


Figure 11

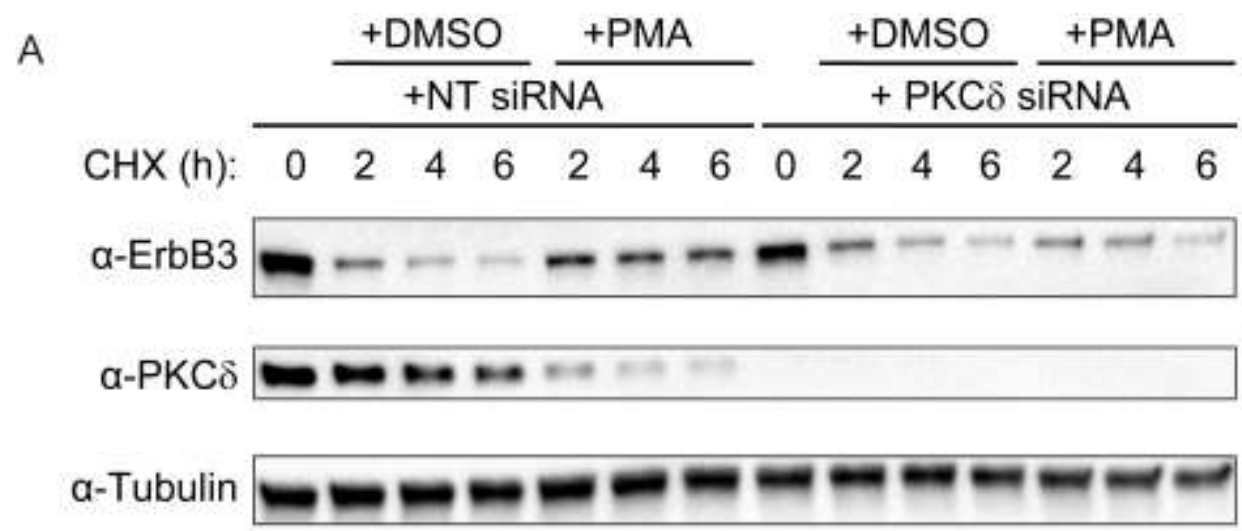

B

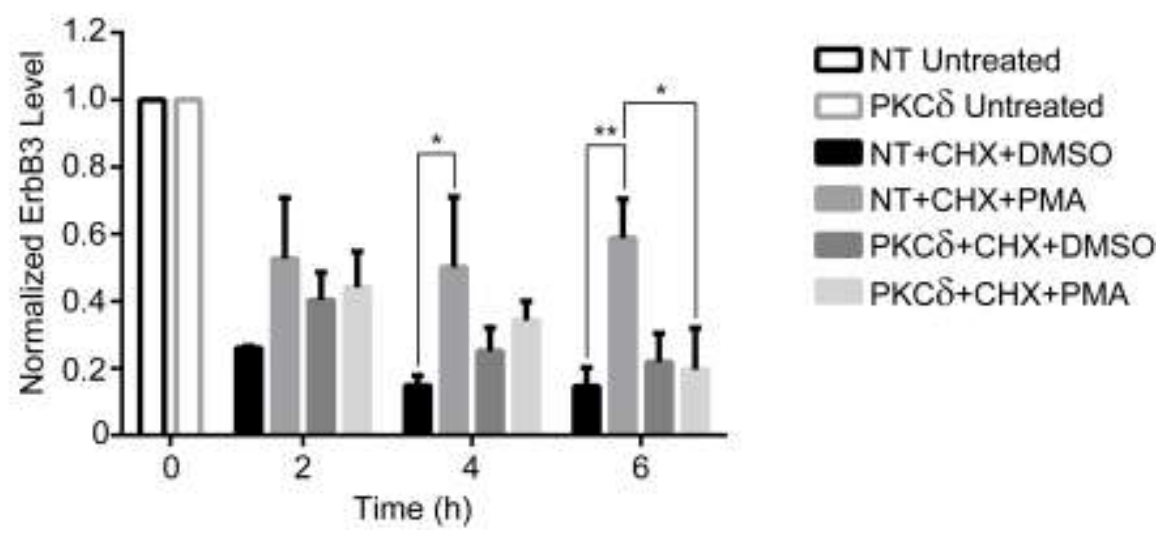

